\title{
The Effects of Ambient Conditions on Helicopter Rotor Source Noise Modeling
}

\author{
Eric Greenwood* \\ NASA Langley Research Center
}

\author{
Fredric H. Schmitz ${ }^{\dagger}$ \\ University of Maryland
}

\begin{abstract}
A new physics-based method called "Fundamental Rotorcraft Acoustic Modeling from Experiments" (FRAME) is used to demonstrate the change in rotor harmonic noise of a helicopter operating at different ambient conditions. FRAME is based upon a non-dimensional representation of the governing acoustic and performance equations of a single rotor helicopter. Measured external noise is used together with parameter identification techniques to develop a model of helicopter external noise that is a hybrid between theory and experiment. The FRAME method is used to evaluate the main rotor harmonic noise of a Bell 206B3 helicopter operating at different altitudes. The variation with altitude of Blade-Vortex Interaction (BVI) noise, known to be a strong function of the helicopter's advance ratio, is dependent upon which definition of airspeed is flown by the pilot. If normal flight procedures are followed and indicated airspeed (IAS) is held constant, the true airspeed (TAS) of the helicopter increases with altitude. This causes an increase in advance ratio and a decrease in the speed of sound which results in large changes to BVI noise levels. Results also show that thickness noise on this helicopter becomes more intense at high altitudes where advancing tip Mach number increases because the speed of sound is decreasing and advance ratio increasing for the same indicated airspeed. These results suggest that existing measurement-based empirically derived helicopter rotor noise source models may give incorrect noise estimates when they are used at conditions where data were not measured and may need to be corrected for mission land-use planning purposes.
\end{abstract}

\section{Notation}

A Rotor Disk Area

$a_{0} \quad$ Ambient Speed of Sound

$a_{x} \quad$ Longitudinal Acceleration of Aircraft

$b \quad$ Number of Rotor Blades

$c_{d_{0}} \quad$ Blade Element Profile Drag Coefficient

$C_{T} \quad$ Thrust Coefficient

$C_{p^{\prime}} \quad$ Acoustic Pressure Coefficient

$C_{p_{i j}}$

$\bar{C}_{L}$

$D_{f}$

$f_{e}$

$g$

$H$

$M$

$M_{A T}$

$M_{H}$

$M_{r}$

$n$

$P$

$p^{\prime}$

Blade Surface Pressure Coefficient

Mean Blade Section Lift Coefficient

Fuselage Parasite Drag

Effective Flag Plate Drag Area

Gravitational Acceleration

Rotor Longitudinal "H-Force"

Section Mach Number

Advancing Tip Mach Number

Hover Tip Mach Number

Surface Normal Direction

Surface Pressure

Acoustic Perturbation Pressure
Mach Number along Propagation Direction

*Research Aerospace Engineer, eric.greenwood@nasa.gov

†Senior Research Professor, fschmitz@eng.umd.edu

Presented at the American Helicopter Society 67th Annual Forum,

Virginia Beach, VA, May 3-5, 2011. This is a work of the U.S.

Government and is not subject to copyright protection in the U.S. $r$

$\bar{r}$

$\mathfrak{r}$

$R$

$R_{*}$

$S$

$t$

$\bar{t}$

$T_{0}$

$U$

V

$v_{i}$

$V_{I A S}$

$v_{n}$

$W$

$x$

$\bar{x}$

$\alpha_{T P P}$

$\Gamma$

$\gamma$

$\gamma_{*}$

$\lambda$

$\mu$

$\xi$

$\rho_{0}$
Lighthill Stress Tensor

Propagation Distance

Non-Dimensional Propagation Distance

Non-Dimensional Radial Station

Rotor Radius

Molar Mass of Air

Blade Surface Area

Time of Observation

Non-Dimensional Time of Observation

Ambient Temperature

Blade Section Velocity relative to Medium

Aircraft Velocity Relative to Medium

Mean Induced Velocity

Indicated Airspeed

Velocity of Medium Normal to Blade Surface

Vehicle Gross Weight

Cartesian Coordinate Vector

Non-Dimensional Cartesian Coordinate Vector

Tip-Path-Plane Angle of Attack

Tip Vortex Circulation Strength

Flight Path Angle

Adiabatic Coefficient of Air

Inflow Ratio

Advance Ratio

Airfoil Surface Slope

Ambient Density 


$\begin{array}{ll}\rho_{S L} & \text { Ambient Density at Sea Level } \\ \sigma & \text { Rotor Solidity } \\ \tau & \text { Time of Emission } \\ \bar{\tau} & \text { Non-Dimensional Time of Emission } \\ \chi & \text { Wake Skew Ratio } \\ \psi & \text { Rotor Azimuth } \\ \Omega & \text { Rotor Rotational Speed }\end{array}$

\section{Introduction}

Helicopter acoustic land-use and mission planning tools are gaining favor for both military and commercial applications. For the military, reducing the detection distance (the distance when an observer first notices the vehicle) is normally the focus. In commercial applications, there is also interest in the detection or noticeability of rotorcraft noise, especially in areas with low ambient noise levels such as rural parks; however, the primary civil focus is designing helicopter operations which reduce community annoyance caused by exposure to helicopter noise. For any of these applications, accurate noise models are needed in order to estimate the acoustic impact of helicopter operations on the observers.

Noise modeling in land-use and mission planning tools is composed of three distinct components: a noise source model, a propagation model, and a receiver model. The noise source model characterizes the far-field noise radiation of the helicopter. The magnitude and direction of rotor noise is strongly dependent on the operating condition of the helicopter, so the external noise radiation must be a function of the helicopter operating state. The propagation model estimates how the sound radiated by the helicopter will propagate through the atmosphere and around terrain to the locations of the observers, and is a strong function of the environmental conditions and terrain. The observer model characterizes the observer characteristics that are important for detection or annoyance.

The focus of this paper is on improving helicopter noise source modeling. Without an accurate description of noise radiated at the source, the acoustic impact of helicopter operations on observers cannot be accurately predicted. Existing empirical noise models are normally based upon acoustic measurements of specific helicopters that are flown in steady-state conditions over a ground-based microphone measurement array. The measured acoustic data are then back-propagated to an assumed point of radiation in order to form a compact helicopter source noise model that is valid at the chosen operating condition of the specific helicopter. This measurement and modeling process is repeated for a number of steady operating conditions, with the acoustic data stored as a function of the specific operating condition. An empirical helicopter noise source model is constructed from this data set which describes the magnitude and direction of radiated noise as a function of the helicopter oper- ating condition. Estimating the noise radiation of this helicopter flying under the measured operating conditions reverses this process and should result in the reproduction of the measured data used to construct the noise source model at that condition.

Several empirical helicopter source noise modeling methods are currently in use. The simplest is derived from simple noise-power-distance extrapolations of measured data at a few microphone locations in order to capture some information about the directivity of helicopter noise. (Refs. 1,2) More complex modeling methods are based on a linear (Refs. 3-5) or planar (Refs. 6, 7) grid of ground based microphones-with the most complex of these methods measuring the radiated noise from maneuvering helicopter in many directions simultaneously using a dense array of microphone positions on the ground. (Ref. 7) All of these modeling approaches have one thing in commonthey are based upon acoustic measurements at one ambient operating condition. Changes in that ambient condition are either not considered or are accounted for indirectly (and perhaps incorrectly) through changes in the other dependent parameters.

Existing land-use and mission planning tools, such as the widely used Rotorcraft Noise Model (RNM), (Refs. 34 ) also neglect the effects of ambient conditions on the helicopter source noise models. The Federal Aviation Administration's Integrated Noise Model (INM) (Refs. 1, 2) does include an empirical correction to data measured during the reference flyover flight condition based on the nondimensional advancing tip Mach number; this is used to adjust the source noise level of the measured flight condition for airspeeds other than that measured, but since the correction is formulated in terms of the non-dimensional advancing tip Mach number, it also includes the effect of temperature changes by way of changes in the ambient speed of sound. However, the simple 2nd order polynomial curve fit used by the INM method does not fully account for the changes in rotorcraft noise sources due to both flight and ambient condition changes, nor can the integrated modeling method capture changes in the directivity of noise due to changes in operating condition. (Ref. 8)

\section{Objective}

The main objective of this paper is to improve the understanding of the effects of ambient conditions on helicopter external noise radiation using a non-dimensional analytical model of main rotor harmonic noise. A new physicsbased experimental method called "Fundamental Rotorcraft Acoustic Modeling from Experiments" (FRAME) is used to assess the acoustic radiation of an example helicopter operating at altitude. The operational, land-use and mission planning implications of ambient conditions on source noise modeling are also briefly addressed. 


\section{Operations at Altitude and the Standard Atmosphere}

Helicopters are strongly influenced by ambient conditions and those conditions are strongly affected by increases in operating altitude. Temperature, density, and ambient pressure all decrease with increasing altitude - this is shown in the top plot of Figure 1 in accordance with the International Standard Atmosphere (ISA) model. (Ref. 9) These changes affect helicopter performance and noise, usually in an adverse manner.

At altitude, the air is thinner and the temperature decreases. Lower air density forces the helicopter to operate at high blade lift coefficients that can decrease performance and increase the likelihood the blade will stall. The lower temperature also increases the operating Mach number of the rotor-again decreasing performance. The aerodynamics of the rotor influence noise radiation. Although the pilot may maintain the same flight condition, as indicated by the aircraft's instruments, the aerodynamic and acoustic state of the rotor will change.

\section{Dimensionally-Defined Flight Conditions}

Flight conditions are typically defined by pilots using dimensional parameters, i.e. indicated airspeed (IAS) and flight path angle. Likewise, these parameters are often used to define the operating condition of the helicopter during the construction and usage of empirical helicopter source noise models. However, for a given indicated airspeed and flight path angle, the non-dimensional parameters that are known to govern rotor harmonic noise vary with ambient density and speed of sound. In this paper, the governing parameters used to define the rotor operating condition are the advance ratio $(\mu)$, wake skew ratio $(\chi)$, thrust coefficient $\left(C_{T}\right)$, and hover tip Mach number $\left(M_{H}\right)$. The definition and physical relevance of these parameters is explained in Appendix [1. The effect of this variation in ambient conditions on the non-dimensional operating condition of a helicopter rotor is illustrated in lower two plots of Figure 1 for a flight condition defined by a constant set of dimensional parametersin particular, for a Bell $206 \mathrm{~B} 3$ operating at a $-6.0^{\circ}$ flight path angle and 60 kts IAS at a variety of ISA altitude conditions.

The variation in governing parameters with ambient conditions leads to a changes in the aerodynamic and acoustic state of the rotor. As air density decreases with increasing altitude, the non-dimensional thrust coefficient increases, bringing the rotor blades closer to stall and increasing the circulation strength of the trailed tip vortices which form the rotor wake. This also leads to an increase in the induced inflow through the rotor. In addition, the decreased air density causes the rotor advance ratio with respect to the medium to increase as true airspeed increases for the same indicated airspeed. This change in advance ratio results in a change in the epicycloidal pattern of the wake; for example Figure 2 shows the "top-view" geometry of the wake for the
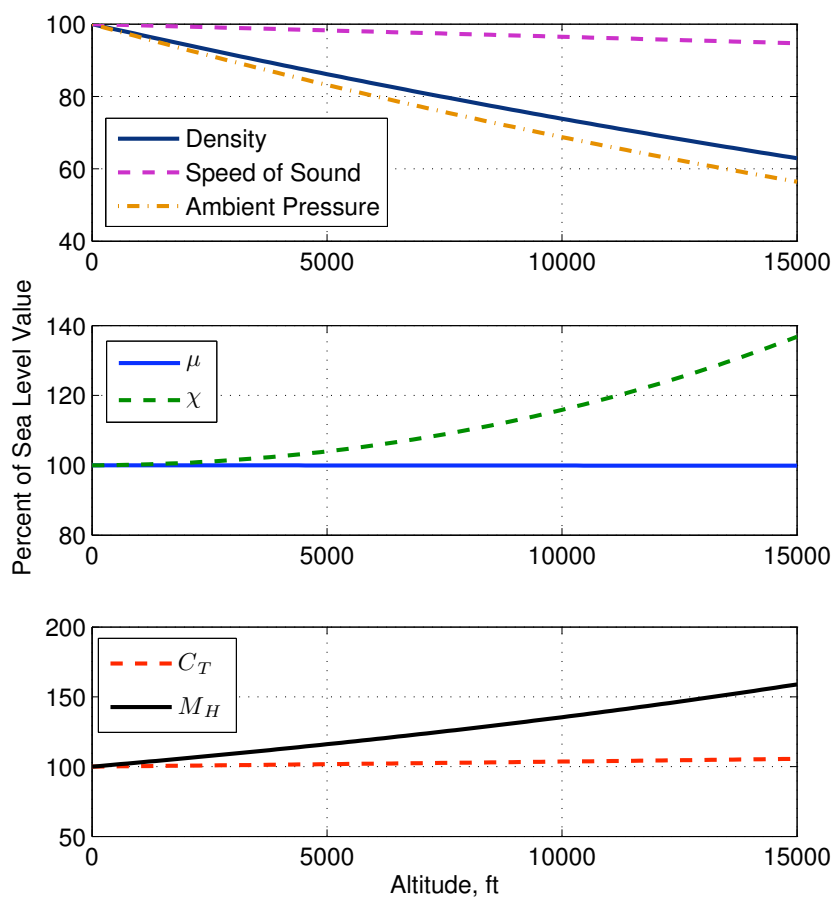

Fig. 1. (top) The variation in atmospheric and governing parameters for the ISA model. (mid/bottom) Corresponding variations in the non-dimensional governing parameters for a constant $60 \mathrm{kts}$ IAS $-6^{\circ}$ FPA approach.

advance ratios associated with $60 \mathrm{kts}$ IAS flight under sea level and at $15,000 \mathrm{ft}$ ISA altitude conditions. While safety of flight considerations dictate that pilots fly the helicopter with respect to indicated airspeed, for noise modeling purposes, true airspeed could be used to define the helicopter flight condition. This is equivalent to holding advanced ratio fixed. The effects of this approach are considered in Appendix II

The increase in the rotor induced inflow with altitude due to the decrease in ambient air density is matched by the increase in advance ratio for the same indicated airspeed. Therefore, the wake skew ratio remains unchanged with altitude for a flight condition maintaining constant indicated airspeed. However, the wake skew ratio will vary with altitude for a constant true airspeed flight condition. The wake skew ratio is related to the average "miss-distance" between the vortices and blades, and is consequently a significant parameter governing Blade-Vortex Interactions (BVI). Lastly, due to the decrease in ambient temperature with altitude, the speed of sound decreases, leading to an increase in the rotor tip Mach number. Altogether, these effects result in a significant change in the rotor acoustic state with variation in altitude that is not accounted for in any of the empirical rotor noise modeling methods currently in use, all of which are developed on the basis of dimensional performance parameters. 


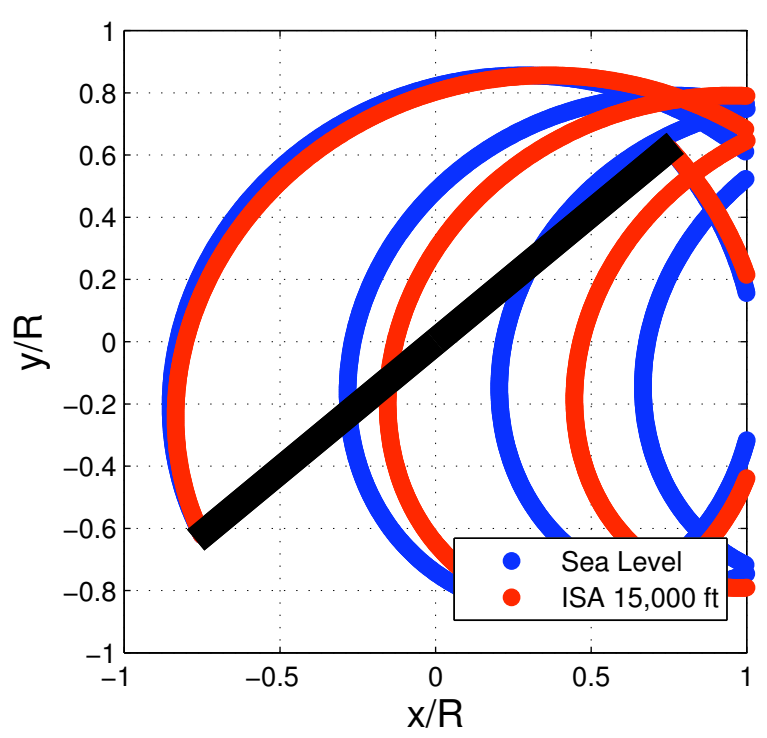

Fig. 2. "Top-view" epicycloidal wake geometry for sea level and ISA 15,000 ft advance ratios at $60 \mathrm{kts}$ IAS.

\section{Fundamental Rotorcraft Acoustic Modeling from Experiments}

The Fundamental Rotorcraft Acoustic Modeling from Experiments (FRAME) methodology (Ref. 10), previously developed by the authors, is used in this paper to describe the external noise radiation of the Bell 206B3 helicopter. FRAME develops non-dimensional semi-empirical noise source models for specific helicopters from measured data. A flowchart of the method is shown in Figure 3. Both wind tunnel and flight test measurements are used in the modeling building process. Wind tunnel measurements allow for more careful control of the operating state of the rotor over a wide range of operating conditions, but are usually limited to scale models of isolated rotors. Flight test measurements are necessary to acquire noise data for the entire full-size vehicle, but for practical reasons the variations in operating condition are limited.

In the FRAME method, both types of experimental measurements of rotor noise are first classified by operating condition in terms of the non-dimensional governing parameters of rotor harmonic noise. For flight test measurements of an entire vehicle, the acoustic signals are transformed to a wind-tunnel reference frame using a time-domain de-Dopplerization technique. (Ref. 11) Periodic averaging is then used to separate the contributions of main rotor, tail rotor, and non-rotor harmonic noise sources from the transformed signal. Using a parameter identification technique, analytical models of the rotor noise sources are then adapted to the acoustic measurements by adjusting a set of physics-based dependent modeling parameters to match the noise radiated for each set of non-dimensional governing parameters. Application of the method across a wide range of operating conditions re-

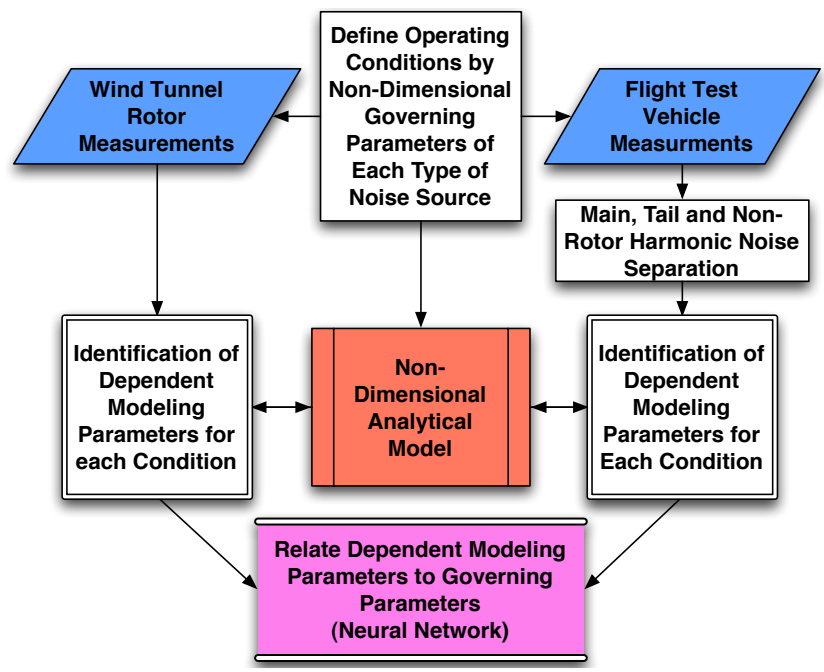

Fig. 3. A flowchart describing the FRAME method for developing rotorcraft source noise models.

sults in a set of dependent modeling parameters associated with the non-dimensional governing parameters of the rotor noise sources. Using the dependent modeling parameters developed from both flight test measurements of full vehicles and wind tunnel measurements of isolated rotors, a neural network model is employed to develop a functional relationship between the non-dimensional governing and dependent modeling parameters over the entire range of operating conditions. By combining the neural network parameter estimator with the associated analytical model, estimates of noise at other operating conditions than those measured may be made. In this paper a FRAME model is constructed for the Bell 206B3 helicopter using a combination of flight test data of the Bell 206B3 (Ref. 12) and wind tunnel data from the similar Operational Loads Survey rotor tested in the German-Dutch Windtunnel (DNW). (Ref. 13)

The underlying analytical framework used in the FRAME model employs a Ffowcs Williams - Hawkings (FW-H) acoustic analogy method. Aerodynamic inputs are provided for each condition using a tunable prescribed wake model combined with an incompressible indicial unsteady aerodynamics model. The non-dimensionalized form of the equation (Eq. 2 in Appendix I) is solved numerically using Farassat Formulation 1A. (Ref. 14) Acoustic sources off the blade surfaces, such as those causing High Speed Impulsive (HSI) noise, are neglected for the moderate advancing tip Mach number range examined in this paper. Thickness noise is directly computed from the blade geometry and rotor operating condition. Loading noise, both lower harmonic and BVI noise, are determined from an assumed aerodynamic model adapted to measured data using parameter identification techniques. The lower harmonic loading variations required to match the measured data are determined directly, but the higher harmonic loading responsible for impulsive BVI noise is found by fitting an adjustable wake model. 
The wake model is based on a modified Beddoes prescribed wake (Refs. 15, 16), where the dependent parameters adjusted by the FRAME method are used to describe the non-uniform longitudinal and lateral inflow variations across the rotor disk, the initial vortex core size and its rate of growth (Ref. 17), the tip vortex rollup radius and the rate of wake contraction (Ref. 18), and the harmonic variation of vortex circulation strength about the rotor azimuth. The velocities induced by the wake onto the rotor blades are then corrected using the Beddoes-Leishman indicial aerodynamics model (Refs. 19, 20) to account for the delayed response of the shed wake on the rapidly changing aerodynamic loading felt by the blade elements. This is similar to the analytical modeling used in previous theoretical research into BVI noise, (Ref.21) but with additional physics-based wake distortion terms to allow the model to be accurately fitted to the measured acoustic data.

Once the fitting process is completed for the entire set of measured data from both the wind tunnel and flight tests, the variations of the dependent parameters with respect to the governing parameters are incorporated into a single artificial neural network model. The result is a single semiempirical model of the Bell 206B3 which is applicable over a wide range of operating conditions defined in terms of the four non-dimensional governing parameters. This model can then be used to generate acoustic hemispheres representing the noise radiated by the rotor for various nondimensionally defined operating conditions, including the effects of ambient condition variations. In this paper noise radiation is described using acoustic hemispheres which show the far-field noise levels normalized to a fixed distance of $30 \mathrm{ft}$ from the main rotor hub. For BVI noise, the levels shown are calculated using the BVISPL metric, which is the unweighted sound pressure level of all main rotor harmonic noise from the 6th through 40th harmonics of the blade passage frequency. For lower harmonic noise, both steady loading and thickness, the unweighted OASPL across the entire audible frequency range is calculated. The resulting acoustic hemispheres are plotted using a Lambert conformal conic projection. (Ref. 22)

\section{Results}

\section{Steady Loading Noise}

First, consider the simple case of a hovering helicopter, where the constant aerodynamic lift is distributed linearly along the blade span, and the corresponding induced drag calculated under the assumption of uniform inflow. Figure 4 shows the OASPL hemisphere representation of the noise radiated by the helicopter at sea level-as expected for steady loading noise in hover, there is no azimuthal directionality to the noise. The OASPL noise metric is used because this noise source is known to be dominated by the fundamental frequency, with noise levels decaying rapidly with higher frequency harmonics. Figure 5 shows the steady

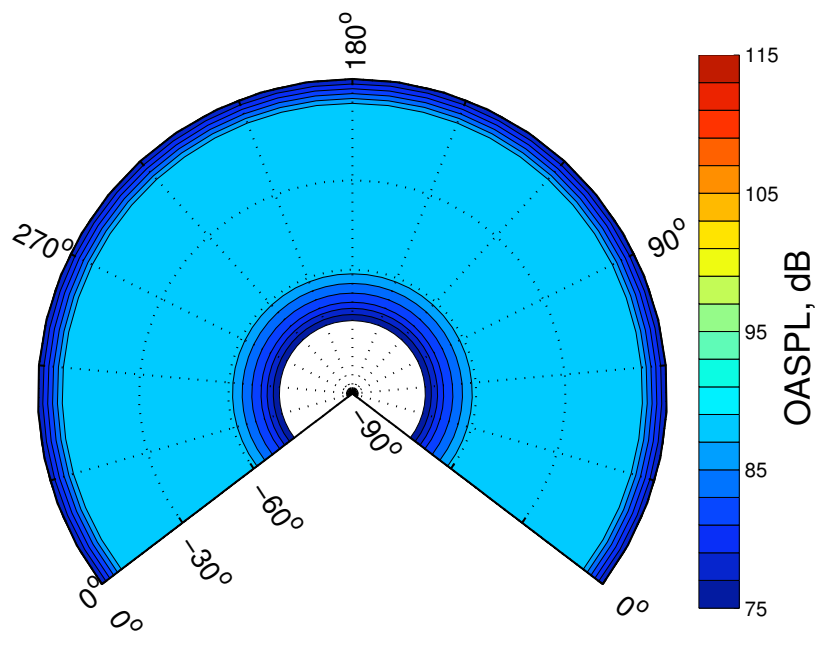

Fig. 4. Hovering flight steady loading noise OASPL hemisphere at ISA sea level conditions.

$\left(C_{T}=0.0029, M_{H}=0.66\right)$

loading noise hemisphere estimated for the $15,000 \mathrm{ft}$ ISA altitude condition, where thrust coefficient has increased for the same vehicle gross weight, due to a decrease in density, and hover tip Mach number has increased for the same rotor rotational rate, due to the decrease in the speed of sound. In addition, the ambient pressure decreases as a function of both ambient speed of sound and density, as per Equation 5 in Appendix I. There is a slight increase in OASPL with altitude, but no change in directivity. The changes in ambient conditions, hover tip Mach number and thrust coefficient are the same in the hover condition as those shown in Figure 1 for forward flight.

Having a non-dimensional analytical model of the rotor harmonic noise sources allows the governing parameter variations to be assessed in isolation from one another, pro-

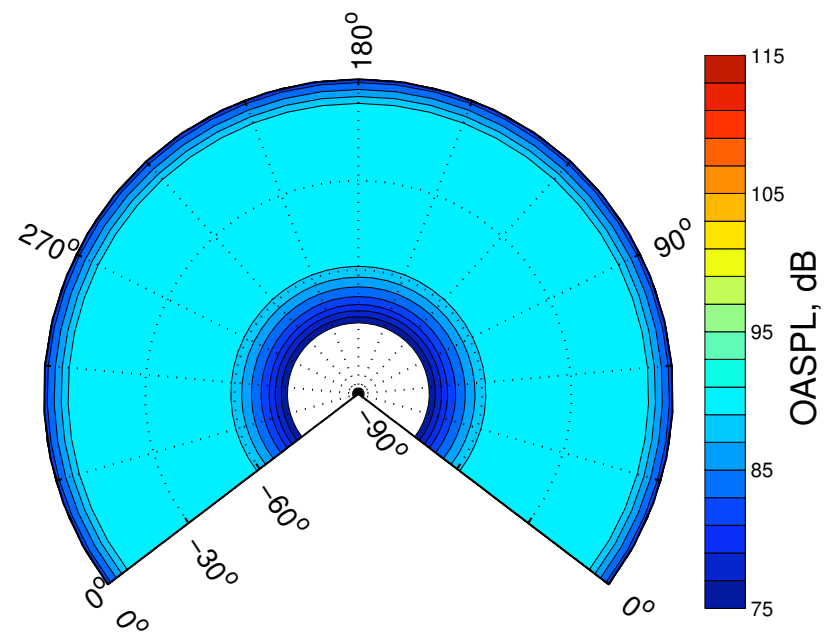

Fig. 5. Hovering flight steady loading noise OASPL hemisphere at ISA $15,000 \mathrm{ft}$ altitude conditions.

$\left(C_{T}=0.0046, M_{H}=0.70\right)$ 


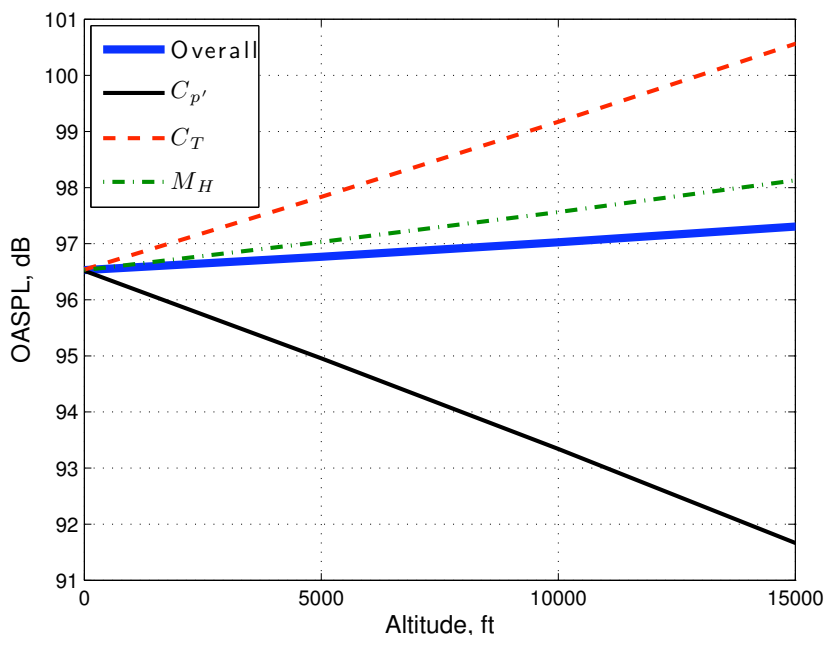

Fig. 6. (a) Variation in steady loading noise OASPL (blue) with ISA altitude conditions. (b) OASPL variations associated with individual governing parameter variations with ISA altitude conditions.

viding some physical insight into the mechanisms which lead to changes in noise radiation. Figure 6 plots in blue the variations in the maximum steady loading noise OASPL radiated in any direction with altitude, for ISA ambient conditions from those associated with sea level to $15,000 \mathrm{ft}$ altitude. In addition, the variations in OASPL are shown for cases where only one parameter is allowed to vary according to ISA conditions, and the rest held fixed at their sea level values. The change in ambient pressure leads to a significant reduction in noise levels when the other parameters, including $C_{T}$, are held fixed. Of course, this is not a physically realizable situation, because the reduction in density leads to a reduction in dynamic pressure, and hence lift; $C_{T}$ must be increased to provide the same thrust at altitude. The increase in $C_{T}$ associated leads to an increase in noise which cancels much of the effect of the reduction in ambient pressure. The increase in hover tip Mach number with altitude leads to a moderate increase in noise levels. In total, there is a small increase in noise with altitude for this simple steady loading source.

\section{Thickness Noise}

Thickness noise, like all other rotor harmonic noise sources, is also affected by changes in ambient conditions. From the non-dimensionalized FW-H equation (Eq. 2 in Appendix I), it is apparent that thickness noise is not governed by parameters that only affect rotor loading, like thrust coefficient and inflow ratio. Therefore, given a description of the rotor geometry, thickness noise can be predicted knowing only the ambient pressure and blade motion through the medium, which is effectively described by the hover tip Mach number and advance ratio. Figure 7 shows the predicted OASPL acoustic hemisphere for thickness noise produced by the Bell 206B3 main rotor during 60kts IAS flight. Indicated

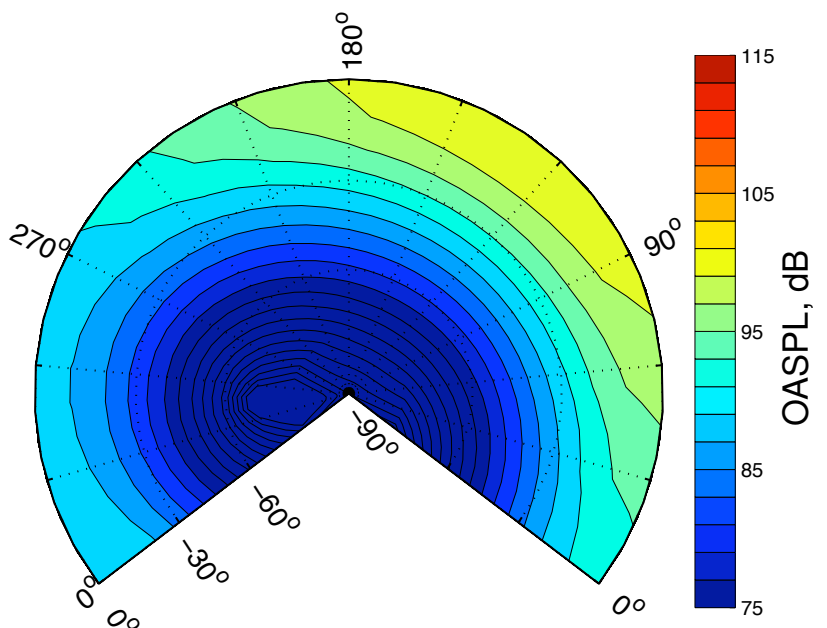

Fig. 7. 60kts IAS OASPL hemisphere of thickness noise at ISA sea level conditions.

$\left(\mu=0.14, M_{H}=0.66, M_{A T}=0.66\right)$

airspeed (IAS) is chosen as an independent parameter in this analysis because it is the airspeed that is normally flown by pilots in order to keep the helicopter within flight safety limits. Thickness noise radiates in-plane ahead of and toward the advancing side of the rotor for any forward flight condition. Likewise, Figure 8 shows the thickness noise hemisphere predicted for the same dimensionally defined flight condition at an ISA 15,000 ft altitude ambient conditions. Predictably, the directivity has not changed substantially, but noise levels have increased. Figure 9 shows the trend in peak thickness noise OASPL with altitude for standard ISA conditions for several different indicated airspeeds. Thickness noise increases more rapidly with increasing altitude for conditions at higher airspeeds.

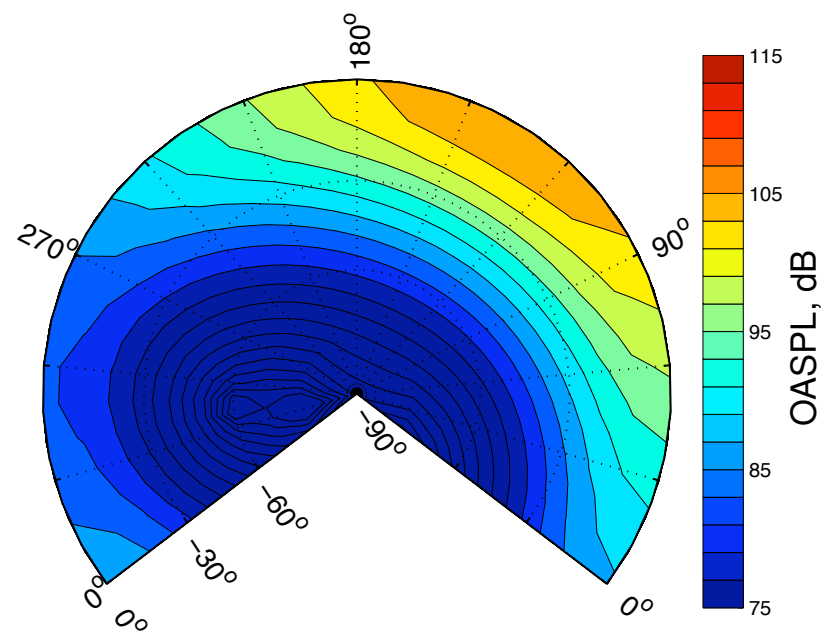

Fig. 8. 60kts IAS OASPL hemisphere of thickness noise at ISA $15,000 \mathrm{ft}$ altitude conditions.

$\left(\mu=0.18, M_{H}=0.70, M_{A T}=0.89\right)$ 


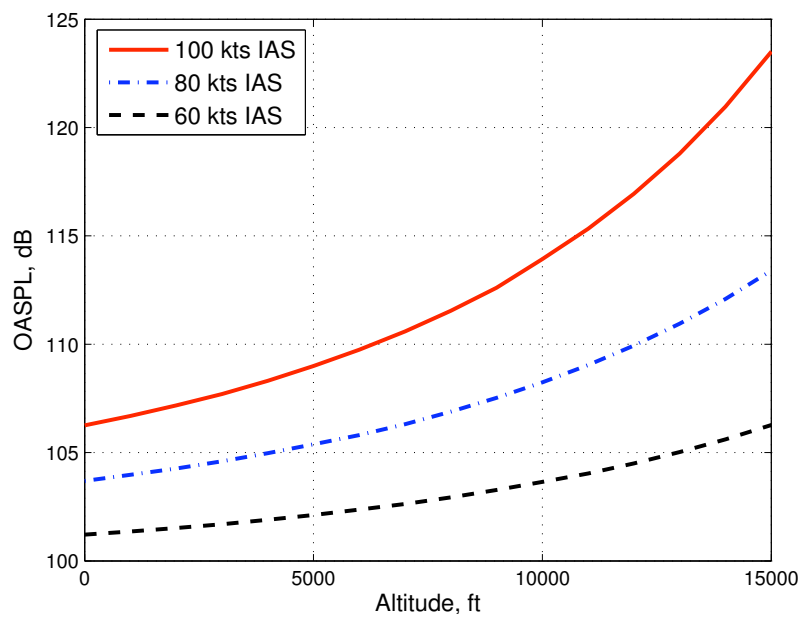

Fig. 9. Peak OASPL thickness noise level variation for ISA altitude conditions for constant IAS flight.

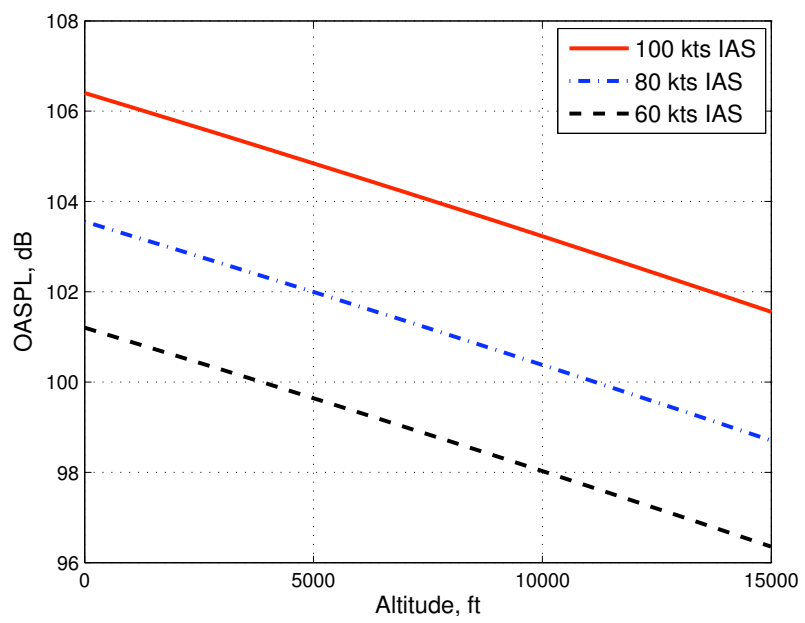

Fig. 10. OASPL variation in thickness noise for ambient pressure variation per ISA altitude conditions.

As for the steady loading case, the contributions of the governing parameters to variation in noise levels can be assessed independently. Figure 10 illustrates the variation in thickness noise due to a decrease in ambient pressure due to altitude, with the sea level values of the advance ratio and hover tip Mach number held fixed. The decrease in ambient pressure leads to a decrease in noise levels, and the effect is proportionate for all cases. (This variation is described by Equation 5 in Appendix I) The variation in thickness noise with only hover tip Mach number varying in accordance to the ISA altitude conditions is shown in Figure 11. As should be expected, the increase in hover tip Mach number with altitude causes a similar increase in thickness noise for all three indicated airspeeds.

Figure 12 shows the variation in thickness noise with only the advance ratio varying in order to maintain the same indicated airspeed (IAS) as density decreases with altitude.

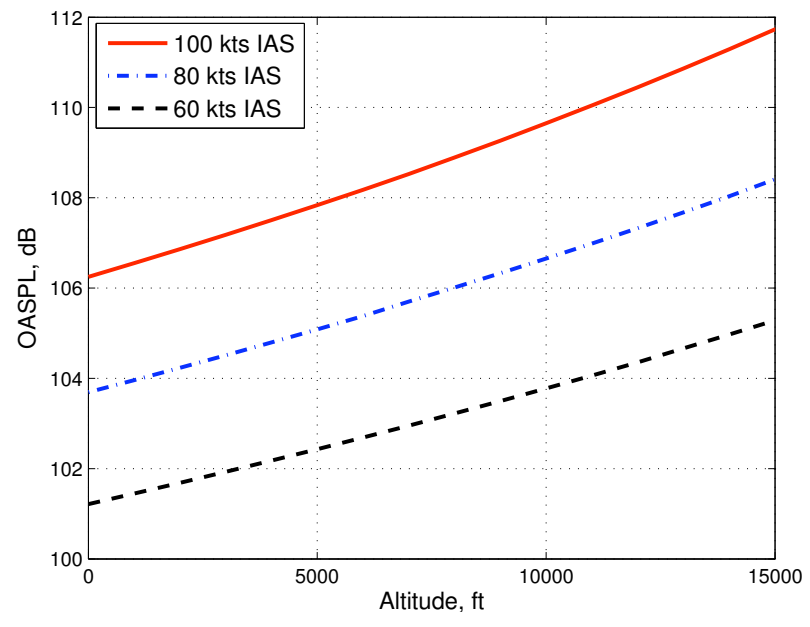

Fig. 11. Thickness noise OASPL trend for hover tip Mach number variation with temperature at altitude.

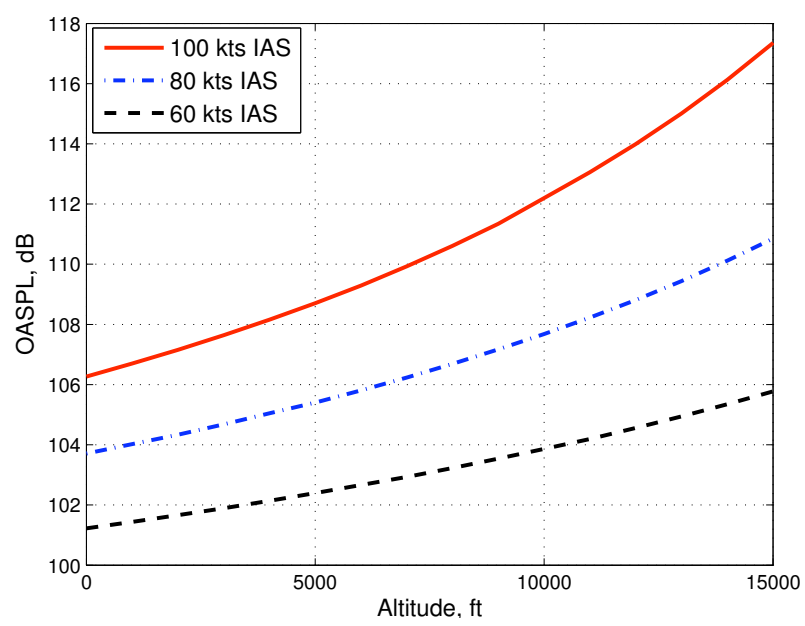

Fig. 12. Thickness noise OASPL trend for advance ratio variation to maintain constant IAS at altitude.

An increase in the advance ratio for the same hover tip Mach number corresponds to an increase in the advancing tip Mach number. (See Equation 11 in Appendix I) The increase in advancing tip Mach number leads to a substantial increase in thickness noise levels. The increase in noise levels with altitude is greater for higher indicated airspeeds. The simple monopole thickness noise calculation used in the FRAME analytical model is known to underpredict noise levels at high advancing tip Mach numbers; the increase in noise with altitude when flying constant indicated airspeed is likely to be even higher in reality than predicted in this paper for high flight speeds.

\section{Blade-Vortex Interaction Noise}

Blade-vortex interaction noise is a special case of loading noise, and is much more complex. The full FRAME 


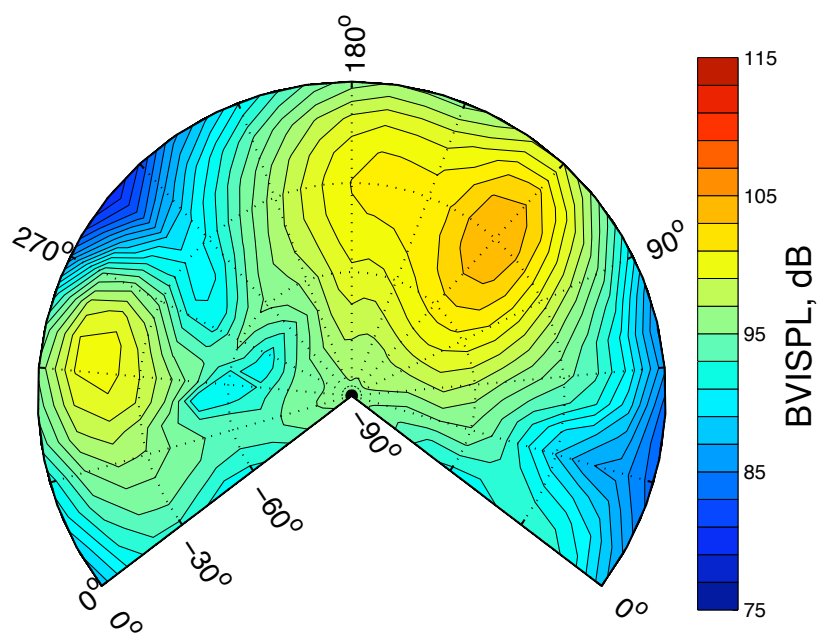

Fig. 13. 60kts IAS $-6^{\circ}$ descent BVISPL hemisphere at ISA sea level conditions.

$\left(\mu=0.14, \chi=0.046, C_{T}=0.0029, M_{H}=0.66\right)$

model described previously is used to show effects of ambient condition variations on BVI noise. Figure 13 shows the BVISPL contours on the surface of a $30 \mathrm{ft}$ radius acoustic hemisphere produced by the Bell 206B3 main rotor FRAME model for a typical $60 \mathrm{kts}$ indicated airspeed (IAS), $-6^{\circ}$ flight path angle approach condition, known for high levels of BVI noise in standard sea level conditions. Two BVI radiate towards the advancing side: the dominant one radiates towards $120^{\circ}$ azimuth and the weaker one towards $160^{\circ}$ azimuth. In addition, a weak BVISPL "hotspot" can be observed on the retreating side of the rotor at $290^{\circ}$ azimuth.

Figure 14 shows a similar BVISPL hemisphere for the same flight condition at ambient conditions corresponding to a 5,000 ft ISA altitude. While all three BVI "hotspots" are still present, the magnitude of the BVI hotspots has increased. The increase in noise levels is not uniform; the noise radiated by the foremost advancing side BVISPL hotspot has increased more rapidly than the others. In addition, the advancing side BVISPL "hotspots" have shifted in direction further towards the advancing side of the rotor.

Figure 15 shows the hemisphere predicted by the model for the same dimensionally defined flight condition at a $10,000 \mathrm{ft}$ ISA altitude. The BVI "hotspot" closer to the retreating side has increased further in level. On the advancing side the foremost "hotspot" has increased even more in BVISPL, dominating the rearmost advancing side "hotspot."

The 15,000 ft ISA altitude BVISPL hemisphere predicted by the FRAME model is shown in Figure 16. BVISPL levels increase even further, but this time it is the advancing side level which increases the most. Both the advancing and retreating side "hotspots" shift rearward.

The retreating side BVISPL levels continue to decrease with further increases in altitude. Figure 17 shows the

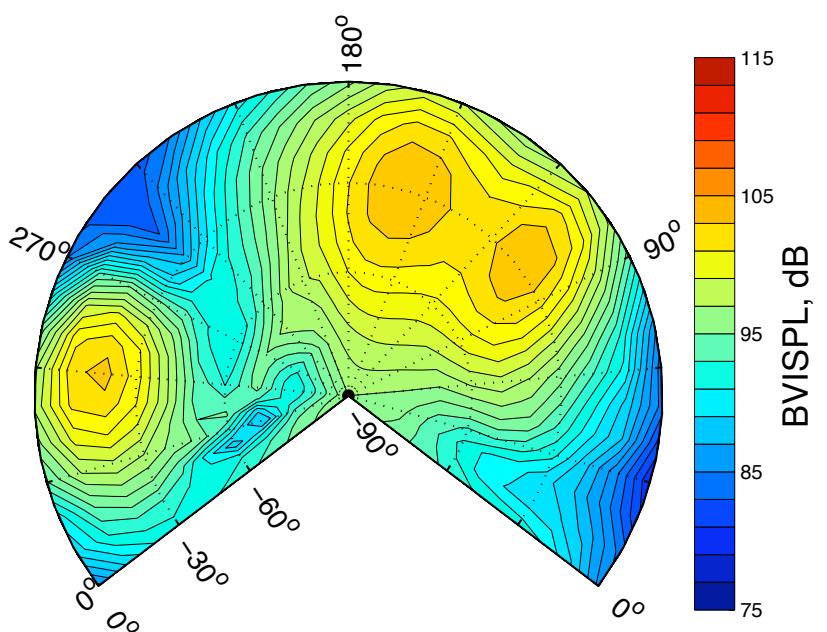

Fig. 14. 60kts IAS $-6^{\circ}$ descent BVISPL hemisphere at ISA 5,000 ft conditions.

$\left(\mu=0.16, \chi=0.046, C_{T}=0.0034, M_{H}=0.67\right)$

$20,000 \mathrm{ft}$ ISA altitude prediction-in this condition, the retreating side BVI "hotspot" has disappeared, but a third advancing side hotspot begins to form ahead of and toward the advancing side of the rotor.

Figure 18 shows the variation of the peak and average BVISPL levels radiated over all directions across the entire range of ISA altitudes. Initially, BVISPL levels decrease with altitude reaching a minimum at about $5,000 \mathrm{ft}$ ISA altitude-after this point, BVISPL see significant increases with altitude throughout the practical range of operating conditions.

Using the non-dimensional model, it is possible to examine in isolation the effect of each of the governing parameter variations with altitude on BVI noise radiation. First, the case is considered where all four non-dimensional gov-

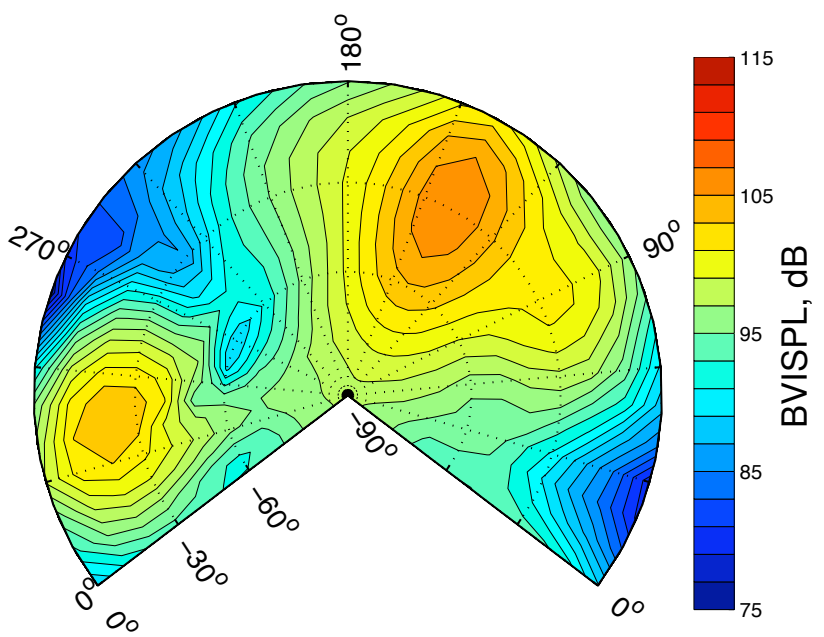

Fig. 15. 60kts IAS $-6^{\circ}$ descent BVISPL hemisphere at ISA 10,000 ft conditions.

$\left(\mu=0.16, \chi=0.046, C_{T}=0.0039, M_{H}=0.68\right)$ 


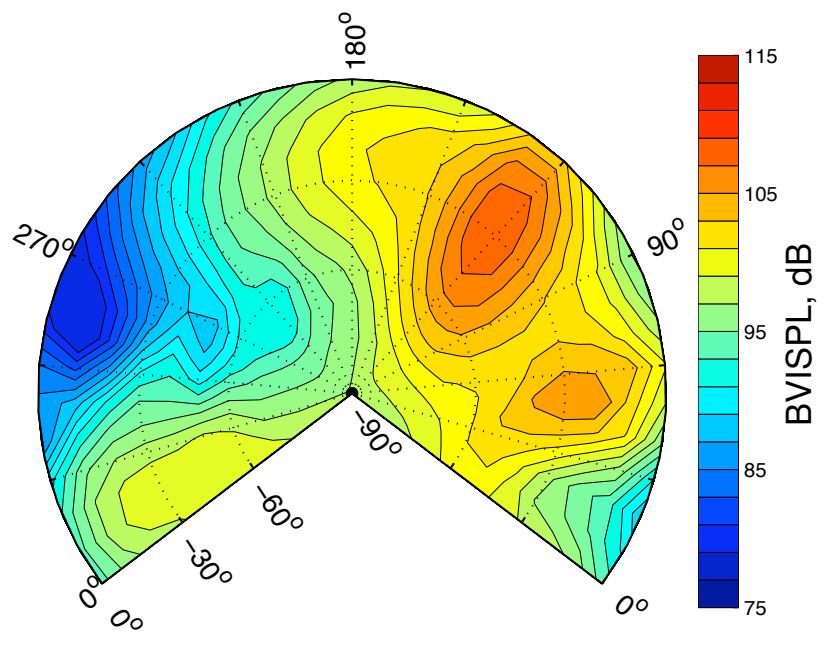

Fig. 16. 60kts IAS $-6^{\circ}$ descent BVISPL hemisphere at ISA 15,000 ft conditions.

$\left(\mu=0.18, \chi=0.046, C_{T}=0.0046, M_{H}=0.70\right)$

erning parameters are held fixed at their ISA sea level values, but ambient pressure is allowed to change. The predicted hemisphere for the $15,000 \mathrm{ft}$ ISA altitude is shown in Figure 19. The directivity of the radiated noise remains unchanged from the sea level case, but the levels have decreased, as would be expected from Equation 5 in Appendix I.

Figure 20 shows the BVISPL hemisphere contours produced for the case where the thrust coefficient $\left(C_{T}\right)$ is increased to the value corresponding to a $15,000 \mathrm{ft}$ ISA altitude (as in Figure 1), but the other three governing parameters, as well as the ambient pressure, are held fixed at their standard sea level values. This has the direct effect of increasing the circulation strength of the trailed vortices in the model, as described in Equation 13 . Consequently, the

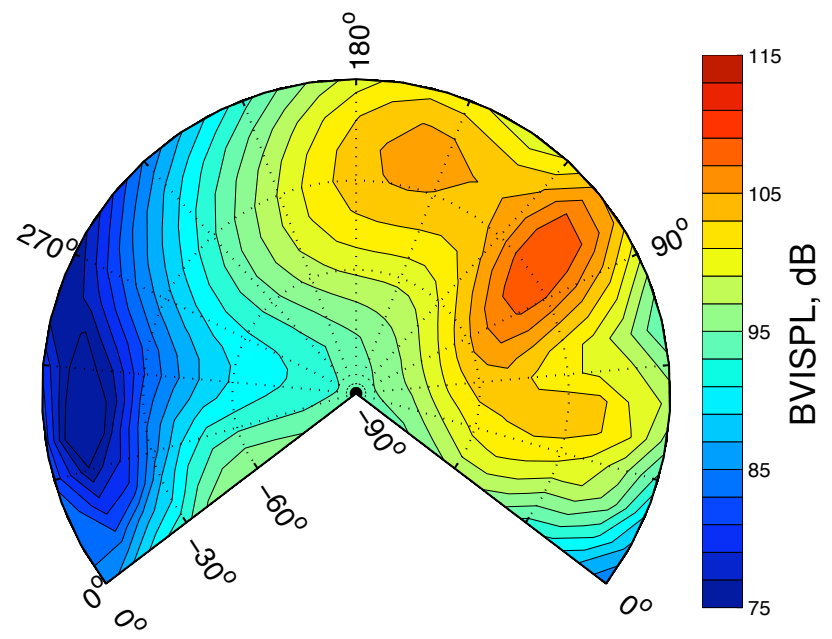

Fig. 17. 60kts IAS $-6^{\circ}$ descent BVISPL hemisphere at ISA $20,000 \mathrm{ft}$ conditions.

$\left(\mu=0.22, \chi=0.046, C_{T}=0.0054, M_{H}=0.71\right)$

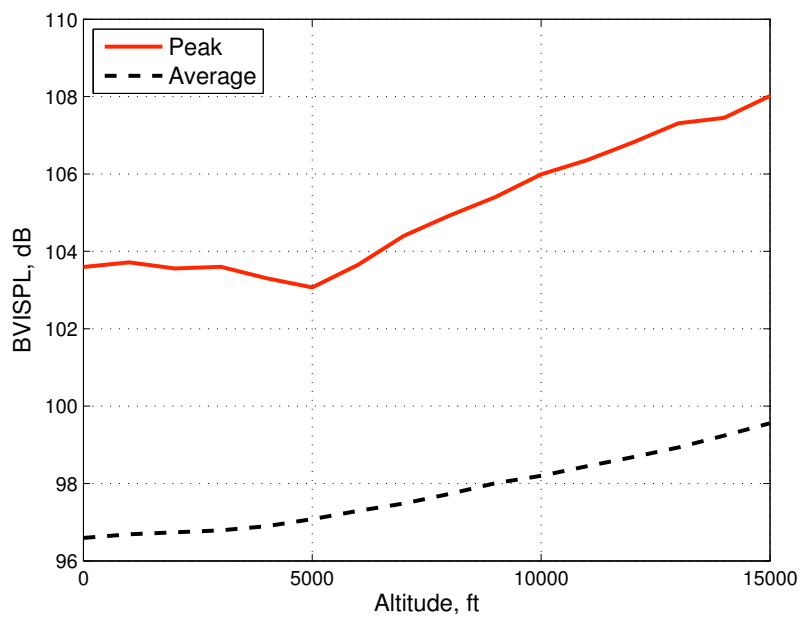

Fig. 18. Variation of BVISPL values with ISA altitude conditions for $60 \mathrm{kts}$ IAS, $-6^{\circ}$ descent flight.

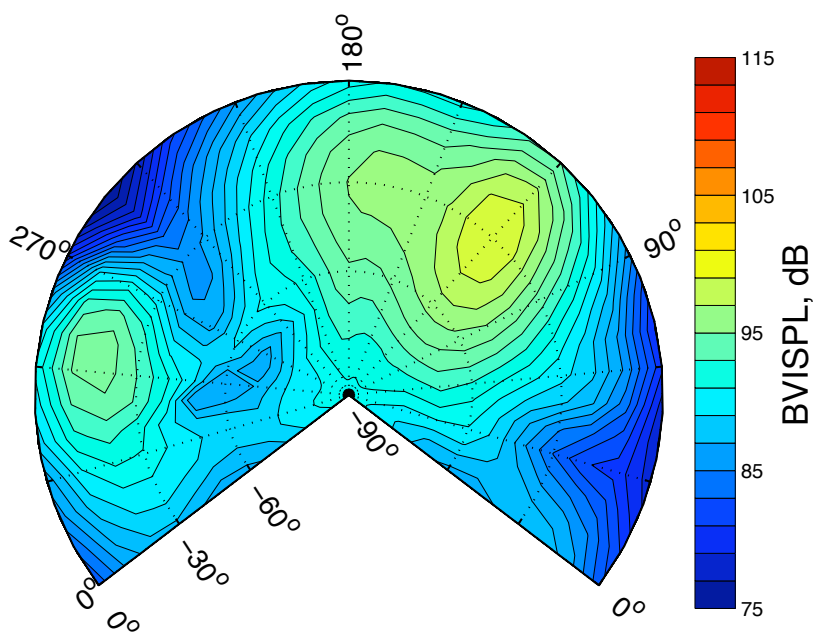

Fig. 19. BVISPL hemisphere at $15,000 \mathrm{ft}$ ISA altitude ambient pressure with $\mu, \chi, C_{T}$ and $M_{H}$ held at ISA sea level values.

noise resulting from each BVI is increased equally resulting in a uniform increase in BVISPL levels in all directions.

Advance ratio increases with increasing altitude for the same indicated airspeed, due to the decrease in air density. Figure 21 shows the resulting BVISPL hemisphere for a change in advance ratio corresponding to $15,000 \mathrm{ft}$ ISA altitude, with the other three governing parameters and ambient pressure held fixed. Compared to BVI noise radiation at standard sea level condition, shown in Figure 13 , the increased advance ratio results in an increase in BVISPL noise levels on the advancing side, due in part to an increase in advancing tip Mach number. In addition, there is a significant change in the directivity of the BVI noise towards the advancing side of the rotor. The increased advance ratio at altitude substantially changes the geometry of the BVI, moving the vortices rearward relative to the blades and changing the interaction angles for the same in- 


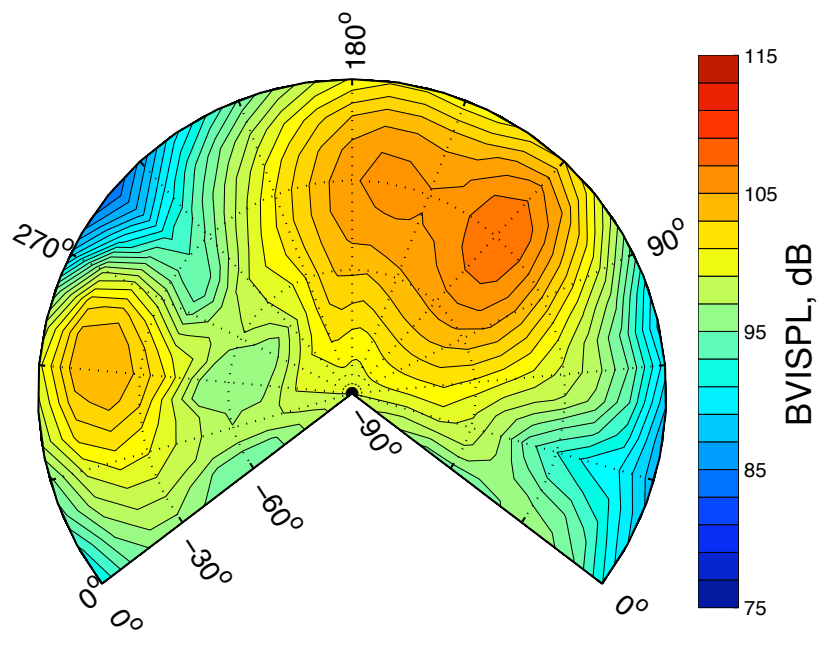

Fig. 20. 60kts IAS $-6^{\circ}$ descent BVISPL hemisphere for $C_{T}$ only at ISA $15,000 \mathrm{ft}$ altitude conditions.

$\left(\mu=0.14, \chi=0.046, C_{T}=0.0046, M_{H}=0.66\right)$

dicated airspeed, as shown in Figure 2 The change in wake geometry influences how the acoustic disturbances of BVI phase in the medium, as explained in Appendix I, and consequently leads to a change in the azimuthal directivity of radiated BVI noise.

As altitude increases, temperature tends to decrease, leading to a reduction in the speed of sound and an increase in all Mach numbers, including the hover tip Mach number. Figure 22 shows the BVISPL hemisphere contours predicted by the model for the 15,000 ft ISA hover tip Mach number, with the other governing parameters and ambient pressure held at their sea level values. In general, the increase in blade section Mach numbers results in an increase in noise levels. The change in hover tip Mach number af-

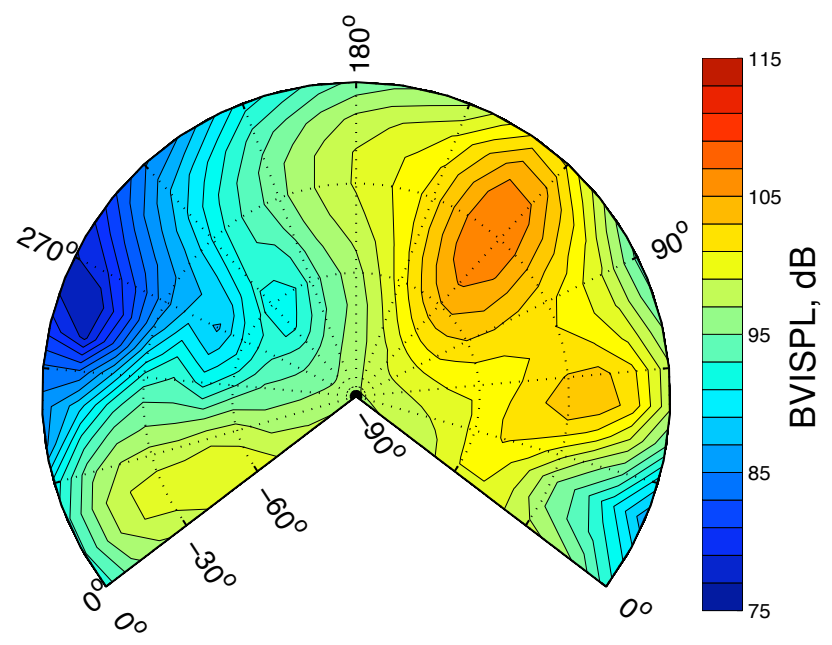

Fig. 21. 60kts IAS $-6^{\circ}$ descent BVISPL hemisphere for $\mu$ only at ISA $15,000 \mathrm{ft}$ altitude conditions.

$\left(\mu=0.18, \chi=0.046, C_{T}=0.0029, M_{H}=0.66\right)$

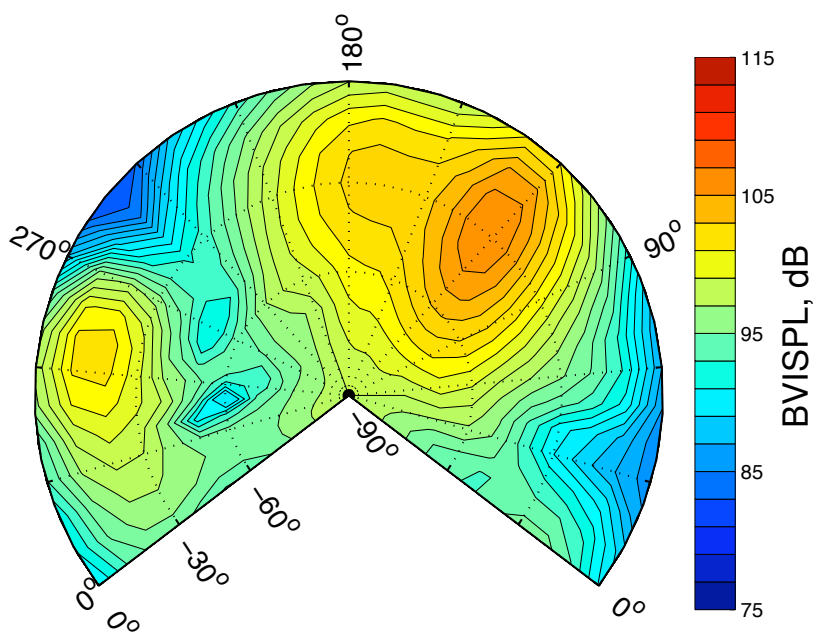

Fig. 22. 60kts IAS $-6^{\circ}$ descent BVISPL hemisphere for $M_{H}$ only at ISA $15,000 \mathrm{ft}$ altitude conditions.

$\left(\mu=0.14, \chi=0.046, C_{T}=0.0029, M_{H}=0.71\right)$

fects all interactions similarly, so that there is no significant change in directivity.

Figure 23 shows the overall trends in BVISPL for variations in each of the governing parameter variations with altitude in isolation. As might be expected, BVISPL levels increase uniformly with the variations in thrust coefficient and hover tip Mach number with ISA altitude conditions. Likewise, the decrease in ambient pressure alone results in a predictable decrease in BVI noise levels. Most notable is the change in BVISPL with advance ratio-initially, BVISPL levels decrease as advance ratio increases. However, after 5,000 ft ISA altitude BVISPL increase with increasing altitude and advance ratio. This is because the change in advance ratio leads to a change the epicyclodial wake geometry. As the BVI locations move aft, the rearmost BVI on the advancing side weakens while the next interaction forward in the wake becomes stronger, as indicated by the difference in BVI noise directivity between BVISPL hemispheres for the sea level (Figure 13) and 15,000 ft ISA (Figure 21) advance ratio operating conditions. At 5,000 ISA altitude, neither interaction is at its strongest and so the overall BVISPL minima is reached. There is no change in the wake skew ratio with altitude for constant indicated airspeed, since the inflow increases in proportion to increases in advance ratio, and so this parameter does not contribute to changes in noise with altitude for this dimensionally-defined flight condition. More details are provided in Appendix I.

Three of the four governing parameters contribute to BVI noise variations with altitude for this flight condition. Variations in hover tip Mach number and thrust coefficient lead to significant increases in BVI noise with increasing altitude, but no significant changes in directivity. This increase is moderated by the reduction in ambient pressure with altitude. Significant changes in the levels and directivity of BVI noise are caused by the variation in advance ratio 


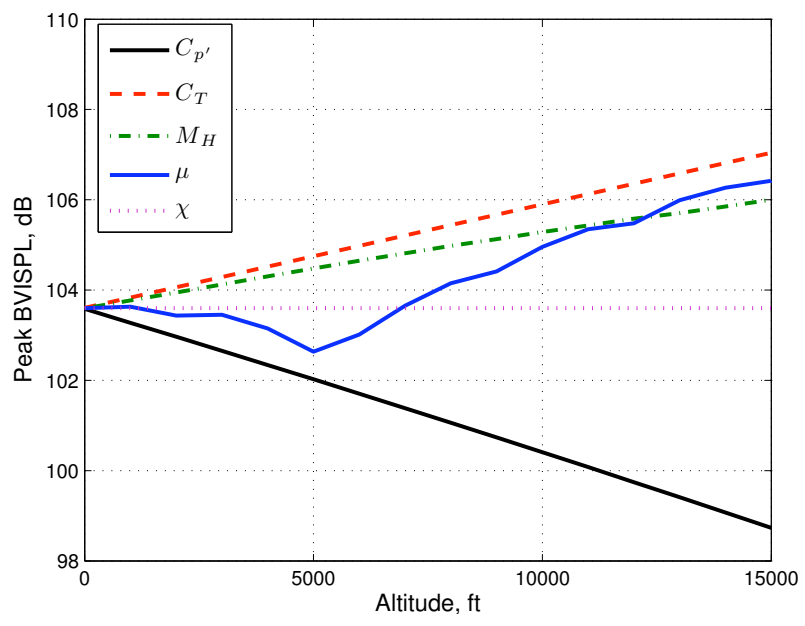

Fig. 23. Peak BVISPL trends for individual governing parameter variations with altitude.

with altitude when indicated airspeed is held constant, due to the corresponding variation in the wake geometry. As different BVI are strengthened or weakened by changes in advance ratio, the peak BVISPL can increase or decrease.

Thus far, the results shown have all been for the same dimensionally defined $60 \mathrm{kts}$ IAS, $-6^{\circ}$ flight path angle approach condition. The trends shown for this flight condition are not necessarily applicable to other flight conditions, where the advance ratio and wake skew ratio define different wake geometries and may result in different variations in noise levels with ambient conditions. Figure 24 shows the trends in peak BVISPL levels with altitude for several different flight conditions. For the faster $80 \mathrm{kts}$ IAS approach, the lowest peak BVISPL levels occur at a higher altitude, due to the change in the epicycloidal wake geometry at a different advance ratio. In addition, because of the initially higher advancing tip Mach number, the rate of increase of BVISPL after this minimum is higher than for the $60 \mathrm{kts}$ IAS case. For the shallower $-3^{\circ}$ flight path angle approach, the BVISPL initially increases with altitudebecause of the greater inflow through the rotor for the shallower descent condition, the wake skew ratio is larger and the BVI near the front of the rotor remains dominant for longer-consequently, the minimum BVISPL point is reached at a higher altitude. For the steeper $-9^{\circ}$ descent, the reduced inflow reduces the wake skew ratio, which keeps the wake near the rotor blades towards the rear of the rotor disk, and the second BVISPL peak occurs earlier as the interaction near the rear of the rotor grows stronger and then weaker with increasing advance ratio.

In addition to changes in ambient conditions due to altitude, temperature changes occurring independently of a change in density result in a change in the speed of sound. This variation causes changes in the hover tip Mach number as well as ambient pressure. Figures 25 and 26 show the BVISPL hemisphere contours for the $60 \mathrm{kts}-6^{\circ}$ flight

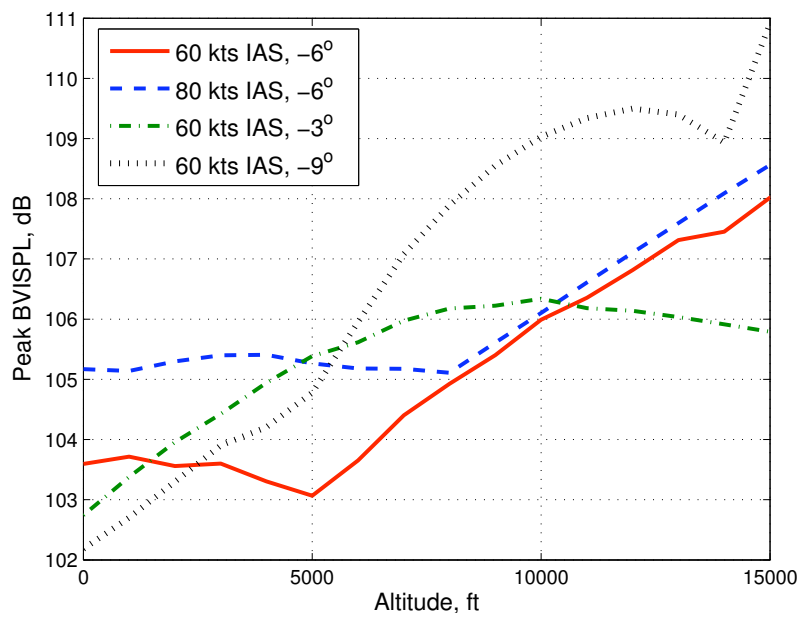

Fig. 24. Peak BVISPL trend for varying ISA altitude conditions at different dimensionally-defined flight conditions.

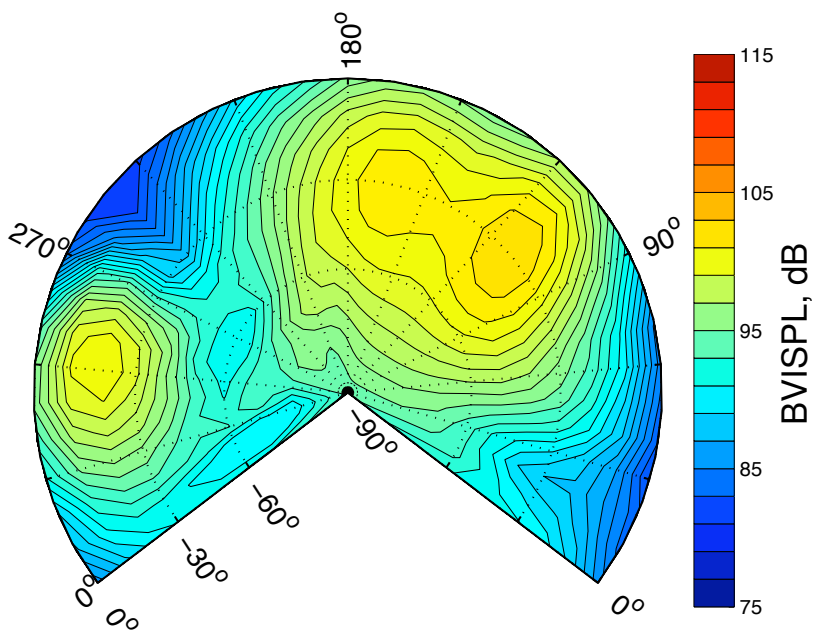

Fig. 25. 60kts IAS $-6^{\circ}$ descent BVISPL hemisphere at ISA sea level $+50{ }^{\circ} \mathrm{F}$ conditions.

path angle approach at sea level ISA $-50^{\circ} \mathrm{F}$ and $+50{ }^{\circ} \mathrm{F}$, respectively. Directivity changes only slightly, with changes in hover tip Mach number having slightly more effect on the advancing side of the rotor. Overall, BVISPL noise levels increase with decreasing temperature and vice versa. Figure 27 shows the overall trend in BVISPL noise levels with changing temperature for this flight condition.

\section{True Airspeed (TAS) as an Independent Parameter}

Advance ratio does not vary with altitude for a fixed true airspeed, but the the wake skew ratio now varies with changing ambient density - this case is examined in more detail in Appendix II Overall, there are smaller, but still significant increases in BVISPL with altitude, due to changes in hover tip Mach number, thrust coefficient, and wake skew ratio. However, because advance ratio remains fixed for a con- 


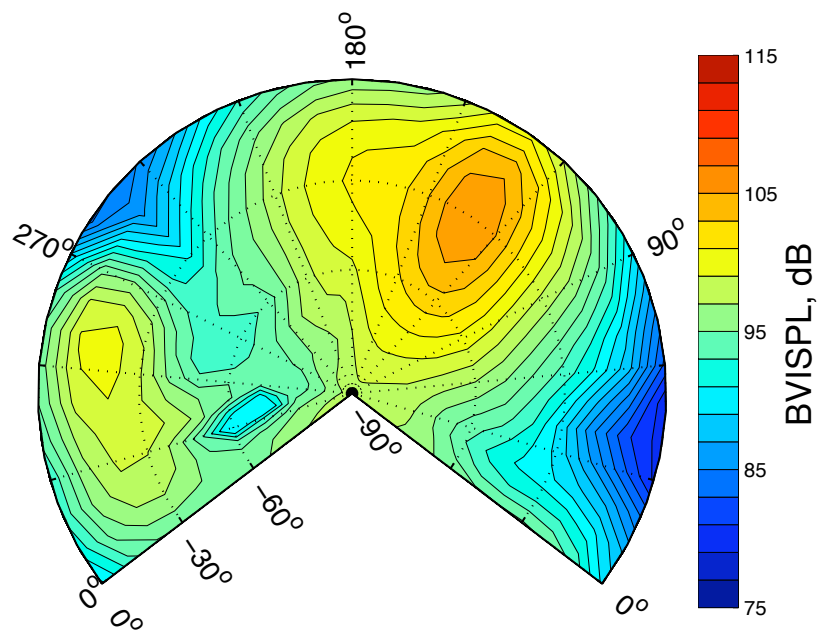

Fig. 26. 60kts IAS $-6^{\circ}$ descent BVISPL hemisphere at ISA sea level $-\mathbf{5 0}{ }^{\circ} \mathrm{F}$ conditions.

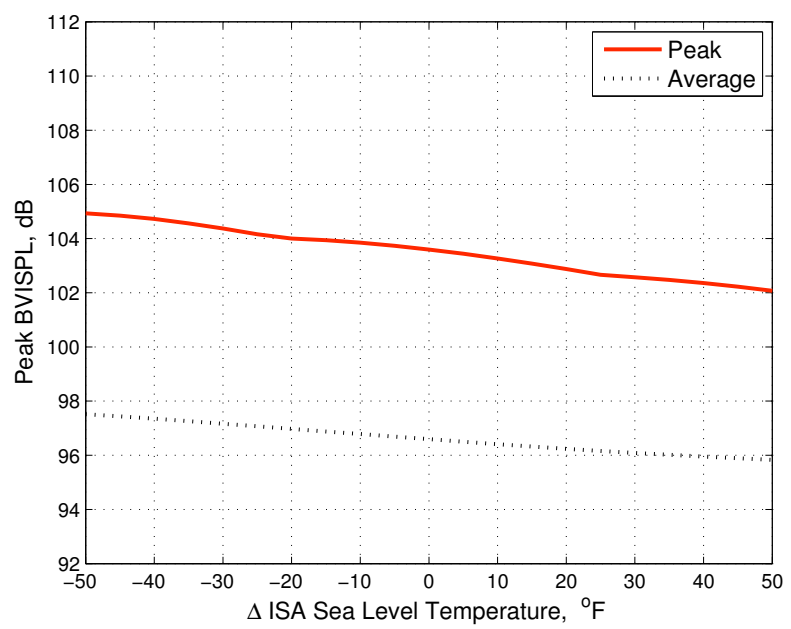

Fig. 27. Variation of BVISPL values for ISA sea level temperature variations at $60 \mathrm{kts}$ IAS, $-6^{\circ}$ descent flight.

stant true airspeed, the directivity of the BVI is not changed significantly. There is a moderate increase in thickness noise levels with altitude for high true airspeed flight conditions and a moderate decrease in levels with altitude for low true airspeed flight conditions. This is because thickness noise is more sensitive to changes in the speed of sound at higher advancing tip Mach numbers.

\section{Implications for Mission Planning Tools}

The implications for the development of land-use and mission planning tools are clear; helicopter source noise models must incorporate the effects of ambient conditions on the rotor noise sources in order to avoid significant errors in the estimation of ground noise and detectability contours. The effects of ambient condition variations are somewhat different for each rotor harmonic noise source; if corrections for ambient conditions are to be developed for empir- ical helicopter source noise modeling methods, the effects on each noise source on the overall external noise radiation need to be considered separately. For this reason, no simple approach is likely to provide an accurate and complete correction of existing helicopter noise source models. Current empirical helicopter noise source models generally classify flight conditions in terms of indicated airspeed and flight path angle - under a single known ambient condition, this corresponds to variations in advance ratio and wake skew ratio. Variations in hover tip Mach numbers and thrust coefficients captured during typical test programs are small and unintentional, but variations in these parameters can be significant over the practical range of helicopter operating conditions. The physics-based and non-dimensional FRAME method offers one solution to this problem, allowing rotor noise models to be constructed for each noise source using both measured flight test data of a full scale vehicle under a practical range of operating conditions and wind tunnel data of similar rotors under a much wider and more carefully controlled range of operating conditions than can be achieved in flight. However, the FRAME method will require validation against measurements of full scale helicopters operating across a range of ambient conditions before it is ready for routine use.

\section{Conclusions}

The parameters that govern helicopter external harmonic noise radiation have been analyzed using a non-dimensional form of semi-empirical theory and parameter identification techniques. Although the approach was applied to the Bell 206B3 two-bladed helicopter in this paper, the findings are thought to be representative of other single main rotor helicopters. Based upon this modeling, the noise produced by a helicopter operating at several different altitudes was estimated. Based upon these results, it was found that:

- In hover, lower frequency noise due to steady loading increases slightly with altitude. Decreases in ambient pressure with altitude reduce the radiated noise but are mitigated by increasing hover Mach numbers. In forward flight, the lower harmonics of loading will contribute as well and noise will also vary with advance ratio.

- Thickness noise levels increase with increasing altitude when flying constant indicated airspeed because of the dependency of thickness noise on advancing tip Mach number. It is mitigated slightly because of decreasing atmospheric pressures, but the strong dependency on advancing tip Mach number dominates ( $8 \mathrm{~dB}$ OASPL/10,000 ft). In practice, increases in advancing tip Mach number may cause HSI noise to develop at altitude, leading to further increases in noise levels and changes in the frequency spectrum of radiated noise. 
- When flying true airspeed, the change in thickness noise levels with increasing altitude is more moderate. The increase in hover tip Mach number increases thickness noise levels, but is counteracted by the decrease in ambient pressure. At high true airspeeds, the net effect is an increase in thickness noise (3/4 dB OASPL/10,000 ft at $100 \mathrm{kts}$ TAS), but at low airspeeds, thickness noise decreases with altitude (-1 dB OASPL/10,000 ft at $60 \mathrm{kts}$ TAS).

- BVI noise can change markedly with altitude for flight operations at constant indicated airspeed. Indicated airspeed compensates for decreasing density at altitude by increasing the forward airspeed of the helicopter. This changes the helicopters true airspeed, which changes the epicycloidal BVI intersection patterns, thus changing the directivity and magnitude of the resulting noise. The decrease in air density with altitude also increases the circulation strength of the tip vortices trailed from each blade, causing increases in BVI noise levels. In addition, decreasing temperature with altitude increases BVI noise radiation levels due to increasing Mach numbers. Changes of up to 7 dB BVISPL per 10,000 feet altitude were estimated.

- Flying true airspeed tends to maintain BVI noise radiation patterns and noise levels. Advance ratio is constant with altitude explaining the similarity of the radiation patterns. Noise levels increase slightly (1 dB BVISPL/10,000 ft) with altitude because of increasing tip vortex strength and increasing Mach numbers, but the increase is mitigated to some degree by the decrease in atmospheric pressure.

- Formulating the problem in non-dimensional terms is helpful in interpreting the variations in the acoustic state of the rotor with variation in the operating condition. The sensitivity of the radiated noise each non-dimensional governing parameter has been clearly shown.

These results show that it is important to consider how measured helicopter acoustic data taken under a given set of conditions might be used to predict noise under different operational conditions. It is obvious that Mach number is an important parameter that will strongly govern radiated noise and should be carefully accounted for. For BVI noise, if the helicopter is flown so that true airspeed (TAS) is constant, then the measured noise patterns that have been gathered at one altitude can approximate the noise that is radiated at other altitudes. However, if indicated airspeed (IAS) is held at these different altitudes, then significant changes in the patterns and levels of BVI noise are to be expected.

\section{Appendix I: Non-Dimensionalization and Development of the Governing Parameters of Rotor Harmonic Noise}

\section{Non-Dimensionalization}

In most cases, physical relationships among variables can be discovered by formulating problems using nondimensional analysis. Buckingham's $\Pi$ theorem, formalized one century ago, provides a systematic procedure for determining a set of non-dimensional parameters governing a physical process. When a problem is correctly formulated on a non-dimensional basis, key relationships between the variables become defined in a way which makes their physical significance more clear.

Consider the Ffowcs Williams - Hawkings (Ref. 23) equation, Eq. 11, which describes the sound generated by arbitrary surfaces in motion:

$$
\begin{aligned}
p^{\prime}(x, t)= & \frac{1}{4 \pi} \frac{\partial}{\partial t} \int_{S}\left[\frac{\rho_{0} v_{n}}{r\left|1-M_{r}\right|}\right]_{\tau} d S-\quad \text { (monopole) } \\
& \frac{1}{4 \pi} \frac{\partial}{\partial x_{i}} \int_{S}\left[\frac{P_{i j} n_{j}}{r\left|1-M_{r}\right|}\right]_{\tau} d S+\quad \text { (dipole) } \\
& \frac{1}{4 \pi} \frac{\partial^{2}}{\partial x_{i} x_{j}} \int_{S}\left[\frac{Q_{i j}}{r\left|1-M_{r}\right|}\right]_{\tau} d S \quad \text { (quadrupole) }
\end{aligned}
$$

The monopole term models thickness noise by considering the rotor blade as a set of monopole mass sources and sinks which describe how the blades displace the medium. The dipole term models the mechanisms of loading noise, including BVI, as a set of aerodynamic dipole sources on the surface of the blades that describe the forces the blades exert on the medium. The quadrupole term includes the effects of complex noise sources inside a fluid volume surrounding the rotor blades - this is how the effect of the transonic flow field that causes HSI noise is modeled. In this paper, the quadrupole term is neglected and the modeling restricted to lower tip Mach number operating conditions where HSI noise does not occur. The FW-H equation for the monopole and dipole terms can be rewritten in non-dimensional form (Eq. 2), following the approach of Reference 13, where all all geometric terms are nondimensionalized by the rotor radius, and all temporal terms by the rotor rotational rate.

$$
\begin{aligned}
C_{p^{\prime}}(\bar{x}, \bar{t})= & \frac{1}{4 \pi} \frac{\partial}{\partial \bar{t}} \int_{S} \frac{\xi M}{\bar{r}\left(1-M_{r}\right)} d \bar{S}- \\
& \frac{1}{4 \pi} \frac{\partial}{\partial \bar{x}_{i}} \int_{S} \frac{C_{p_{i j}} n_{j} M^{2}}{\bar{r}\left(1-M_{r}\right)} d \bar{S}
\end{aligned}
$$

where the acoustic pressure has been non-dimensionalized with respect to the ambient pressure (expressed as a function of ambient density and speed of sound):

$$
C_{p^{\prime}}(\bar{x}, \bar{t})=\frac{p^{\prime}(\bar{x}, \bar{t})}{\rho_{0} a_{0}^{2}}
$$


and the blade surface pressures are non-dimensionalized by the dynamic pressure at the respective blade element:

$$
C_{p_{i j}}=\frac{p_{i j}}{\rho_{0} U^{2}(\mathfrak{r}, \psi)}
$$

This non-dimensionalization indicates that for an otherwise identical non-dimensional operating condition of the rotor, the acoustic pressure amplitudes will vary in proportion to the ambient pressure ratio, as expressed in Eq. 5 .

$$
p_{1}^{\prime}(\bar{x}, \bar{t})=\frac{\left(\rho_{0} a_{0}^{2}\right)_{1}}{\left(\rho_{0} a_{0}^{2}\right)_{2}} p_{2}^{\prime}(\bar{x}, \bar{t})
$$

\section{Governing Parameters of Rotor Harmonic Noise}

The non-dimensional rotor operating condition is defined by a set of four independent parameters which are known (Refs. 13, 24) to govern the rotor harmonic noise sources, and these parameters can be expressed as the wake skew ratio $(\chi)$, advance ratio $(\mu)$, thrust coefficient $\left(C_{T}\right)$, and hover tip Mach number $\left(M_{H}\right)$. This set of four non-dimensional governing parameters is derived from the physical processes of rotor harmonic noise generation, including thickness and BVI noise.

For a fixed hover tip speed, hover tip Mach number is defined by the ambient speed of sound:

$$
M_{H}=\frac{\Omega R}{a_{0}}
$$

where ambient speed of sound can be estimated in air with a function of ambient temperature:

$$
a_{0}=\sqrt{\gamma_{*} R_{*} T_{0}}
$$

Likewise, the rotor advance ratio is determined by the true airspeed at which the rotor moves through the medium.

$$
\mu=\frac{V}{\Omega R}
$$

This can be related to the indicated airspeed, which is a function of dynamic pressure, through air density using the following expression valid for the range of rotorcraft airspeeds and altitudes:

$$
V_{I A S}=V \sqrt{\frac{\rho_{0}}{\rho_{S L}}}
$$

The combination of hover tip Mach number and advance ratio therefore specify the Mach number of all blade sections at all azimuths.

$$
M=M(\mathfrak{r}, \psi)=M_{H}(\mathfrak{r}+\mu \sin \psi)
$$

For example, equation 10 can be used to relate the hover and advancing tip Mach numbers:

$$
M_{A T}=M_{H}(1+\mu)
$$

The advance ratio and hover tip Mach number also set the epicycloidal pattern of the wake formed by the trailed tip vortices responsible for BVI, as shown in Figure 28. In combination, these two governing parameters set the number of potential BVI occurring on the advancing and retreating sides of the rotor, as well as the interaction angles between the rotor blades and the vortices during BVI events. The interaction angle of BVI controls how the acoustic disturbance accumulates in phase through the medium, and therefore contributes to the amplitude of BVI impulses and determines the azimuthal directivity of BVI noise. Figures 29 and 30 use 2D Huygens' wavelets to illustrate how the BVI phasing process causes the radiation of noise towards specific azimuths for oblique and parallel BVI, respectively.

The thrust coefficient is the non-dimensionalization of rotor thrust with respect to a reference dynamic pressure, calculated from the rotor tip speed and ambient air density, and a reference area taken as the rotor disk area.

$$
C_{T}=\frac{T}{\rho_{0} A(\Omega R)^{2}}
$$

For steady flight conditions, the rotor thrust is approximately equal to the vehicle weight. Therefore, the ambient air density determines thrust coefficient for a particular rotorcraft with fixed gross weight and rotor tip speed. The thrust coefficient relates to the blade section loading, and hence influences lower harmonic loading noise. In addition, the trailed tip vortex circulation strength is directly proportional to rotor thrust coefficient, which influences the strength and acoustic impact of BVI events. For instance, the analytical solution for the non-dimensionalized trailed tip vortex circulation strength due to an idealized triangular spanwise lift distribution is shown in Eq. 13 .

$$
\frac{\Gamma}{\Omega R^{2}}=\frac{2 \pi C_{T}}{b}
$$

The thrust coefficient is also directly related to the blade loading coefficient, $C_{T} / \sigma$, which has a similar form to the non-dimensionalized blade surface pressure term, $C_{p_{i j}}$, of Equation 2 Through Equation 14, provided in Reference 26, the blade loading coefficient can also be related to the mean lift coefficient of the rotor blade sections. In hovering flight, rotor stall occurs for $C_{T} / \sigma \sim 0.13$, and decreases with increasing advance ratio due to asymmetry in local lift coefficient. Consequently, as altitude increases so does the blade loading coefficient, bringing the rotor closer to stall. Not only does this impose limits on the operation of the helicopter in high altitude conditions, it will also change rotor's aerodynamic and acoustic state. 


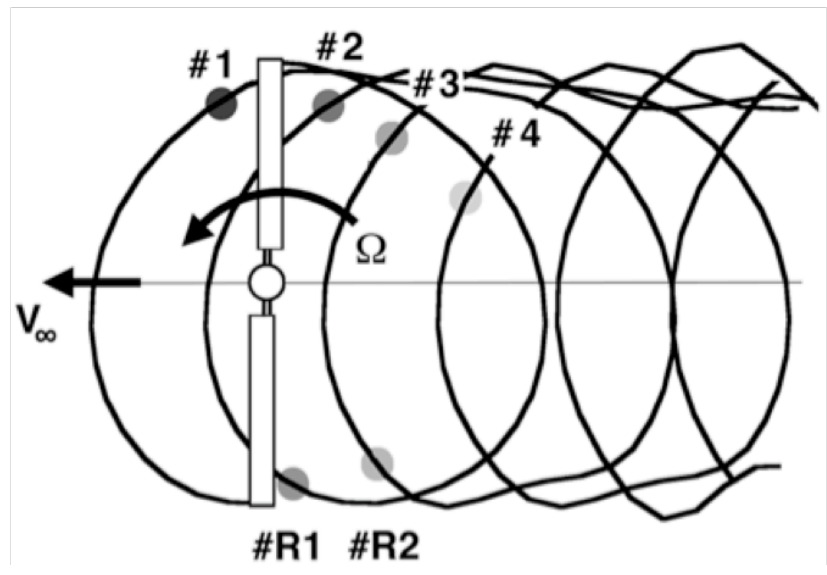

Fig. 28. The top-view geometry of the wake is set by the rotor advance ratio and hover tip Mach number and determines the BVI locations and interaction angles. From Reference 25 .

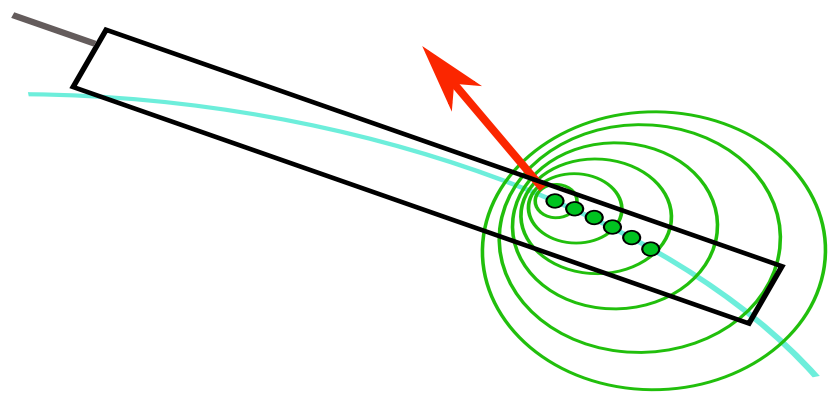

Fig. 29. Huygens' wavelet diagram of an oblique BVI, as in interaction \#2 of Figure 28.

$$
\frac{C_{T}}{\sigma}=\frac{\bar{C}_{L}}{6}\left(\frac{1-\mu^{2}+9 \mu^{4} / 4}{1+3 \mu^{2} / 2}\right)
$$

The wake skew ratio, $\chi$, is defined in this paper to be the ratio of net rotor inflow perpendicular to the rotor tippath-plane to flow parallel to the tip-path-path. The can be expressed as the ratio of the non-dimensional inflow ratio to the advance ratio:

$$
\chi=\frac{\lambda}{\mu}
$$

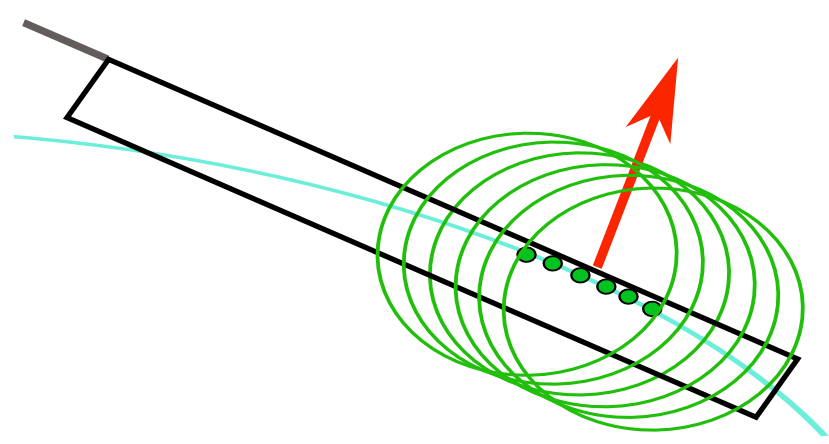

Fig. 30. Huygens' wavelet diagram of a parallel BVI, as in interaction \#3 of Figure 28 . where the rotor inflow ratio is the mean inflow velocity through the rotor disk non-dimensionalized by the rotor tip speed, and is defined:

$$
\lambda=\frac{-V \sin \alpha_{T P P}+v_{i}}{\Omega R}
$$

The inflow ratio is determined by both the freestream velocity through the rotor and the velocity induced by thrust. The contribution of the induced velocity can be estimated using simple momentum theory, resulting in Eq. 17

$$
\lambda=-\mu \tan \alpha_{T P P}+\frac{C_{T}}{2 \sqrt{\lambda^{2}+\mu^{2}}}
$$

The component of the freestream velocity which passes through the rotor and contributes to the inflow ratio is determined by the angle of attack between the rotor tip-pathplane and the free stream velocity. This angle of attack can be determined to first order using a simple longitudinal force balance:

$$
\alpha_{T P P}=-\frac{D_{f}+H}{W}-\gamma-\frac{a_{x}}{g}
$$

The tip-path-plane angle of attack depends on the flight path angle and longitudinal acceleration of the helicopter. In addition, the fuselage parasite drag and rotor $\mathrm{H}$-force contribute to the tip-path-plane angle of attack. For steady flight conditions where thrust and weight can be assumed equal, both the drag-to-weight (Eq. 19) and H-force-toweight (Eq. 20) ratios can be estimated in terms of advance ratio and thrust coefficient.

$$
\begin{gathered}
\frac{D_{f}}{W}=\frac{f_{e}}{2 A C_{T}} \mu^{2} \\
\frac{H}{W}=\frac{\sigma c_{d 0}}{8} \frac{1+4.6 \mu^{2}}{\mu C_{T}}
\end{gathered}
$$

The wake skew ratio generally determines how at which angle the the trailed tip vortices composing the rotor wake will convect from the front of the rotor tip-path-plane, and consequently governs the average "miss distance" between the blades and vortices during BVI events, as shown in Figure 31. This average "miss distance" is an aggregate measure of the separation of the rotor wake from the rotor blades, and is not directly associated with any single vortex; for instance, when the average "miss distance" is small (i.e. the wake is near the rotor) changes in the mean inflow ratio may result in the separation between the blades and the vortices at some locations on the rotor to increase while at other locations the separation distance decreases. As the separation distance between the vortices and blades decreases, the effect of the trailed vortex on the blade loading increases; consequently, the "miss distance" has a significant effect on BVI noise. In general, "miss distances" are at a minimum, and BVI at a maximum, when the wake skew ratio is near zero, and the vortices stay near the rotor tip-path-plane as they convect to the rear of the rotor. 


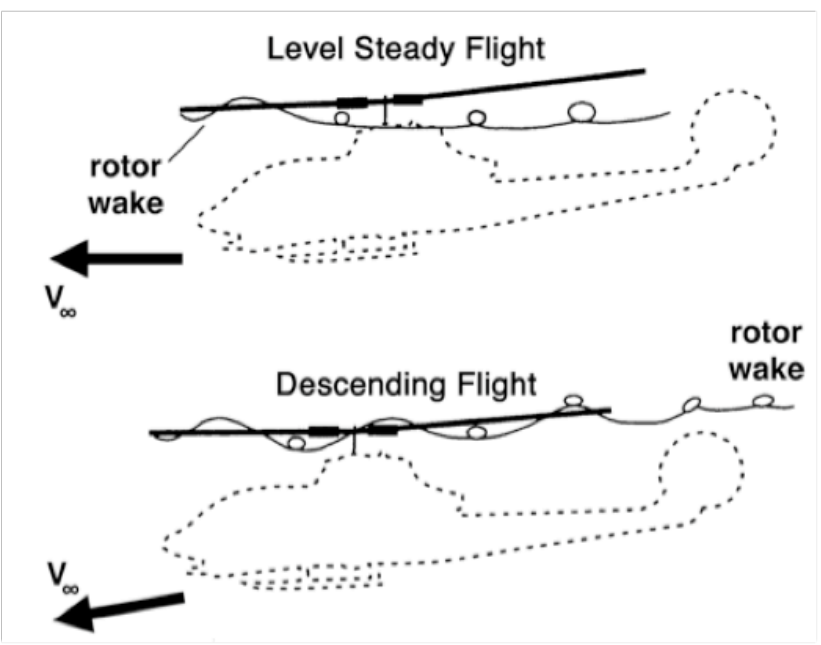

Fig. 31. The side-view geometry of the wake is set by the rotor inflow and determines the "miss-distance" between the vorticies and blades during BVI. From Reference 25 .

\section{Appendix II: Flight Conditions Defined by True Airspeed}

While flight conditions are typically defined in terms of indicated airspeed (IAS), which varies with dynamic pressure, another option is to define flight conditions with respect to true air speed (TAS). Figure 32 shows the variation in non-dimensional governing parameter values with changing ISA altitude conditions for the case where true airspeed is held constant, similar to Figure 1 for the typical indicated airspeed case. In this situation, the advance ratio does not vary, such that the freestream dynamic pressure decreases with altitude. This leads to a decreasing fuselage parasite drag and rotor $\mathrm{H}$-force, causing the rotor tip-pathplane to tilt backwards and reducing the inflow through the rotor. On the other hand, the reduction in air density leads to a substantial increase in induced inflow-overall, there is a greater increase in inflow for constant true airspeed (TAS) than when flying constant indicated airspeed (IAS). Since inflow increases relative to the constant true airspeed, the wake skew ratio varies with altitude. This results in the descent angle required for zero wake skew (and hence near maximum BVI noise) varying with altitude, as shown in Figure 33 Depending on the true airspeed flow, the sensitivity of BVI to flight condition will change. The BVISPL hemisphere contours for a $60 \mathrm{kts}$ TAS $-6^{\circ}$ flight path angle approach condition at ISA altitudes of 5,000, 10,000 and 15,000 ft are shown in Figures 34, 35 and 36, respectively. The retreating side BVI "hotspot" location remains fixed with altitude, since advance ratio remains unchanged. The increase in the wake skew ratio causes a small increase in the "miss-distances" of the BVI, but this is mitigated by the increase in thrust coefficient and Mach numbers with altitude. The overall increase in BVISPL with altitude is shown in Figure 37. In general, the changes in BVISPL magnitude
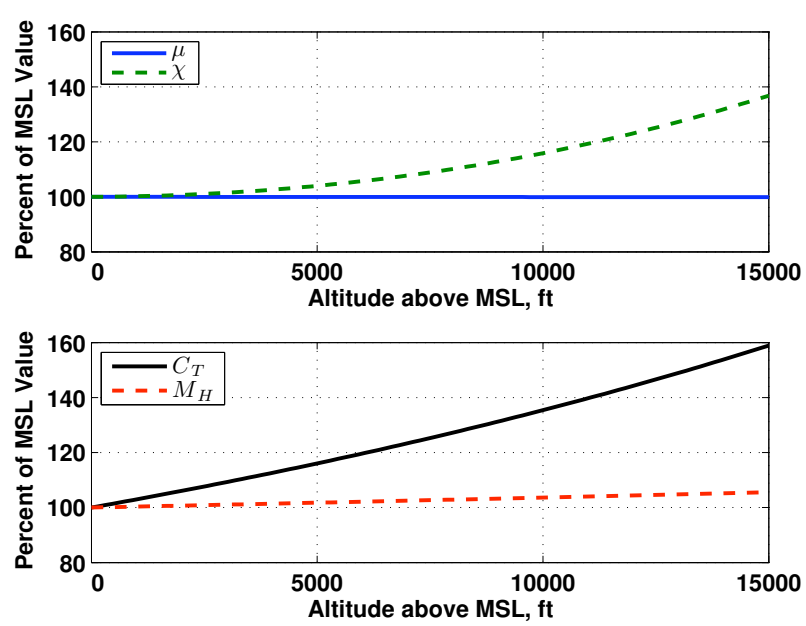

Fig. 32. The relative variation in non-dimensional governing parameters with ISA altitude for a Bell $206 \mathrm{~B} 3$ in 60 kts TAS $-6.0^{\circ}$ descending flight.

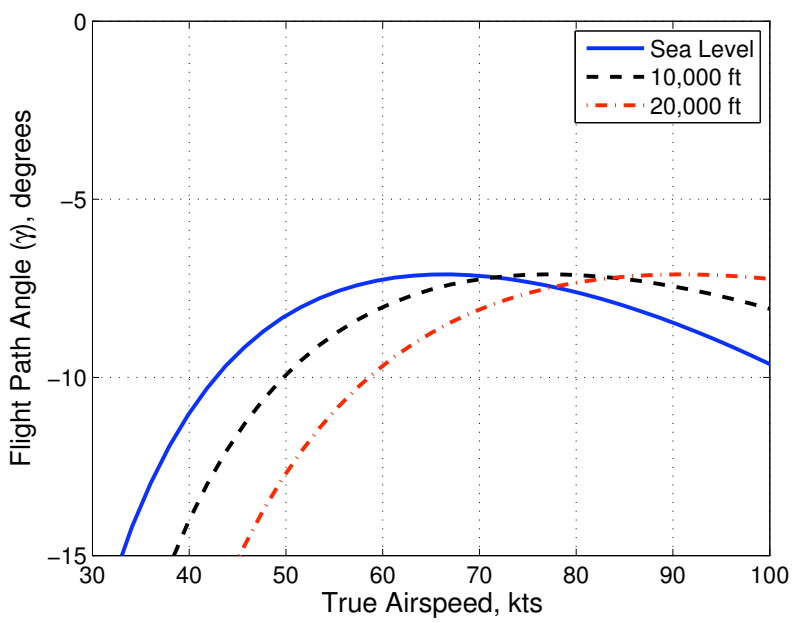

Fig. 33. A plot of the dimensionally-defined zero wake skew ratio flight conditions at various altitudes.

and directivity with changing ambient conditions are somewhat less pronounced and are more predictable when defining flight conditions by true airspeed instead of indicated airspeed, because the advance ratio remains fixed.

The variation in thickness noise with altitude is likewise different for flight conditions defined by true airspeed than for those defined by indicated airspeed. Since advance ratio remains fixed, the advancing tip Mach number only increases in proportion to the increase in hover tip Mach number with altitude. In addition, the reduction in ambient pressure with altitude reduces the amplitude of thickness noise. Figure 38 plots the peak thickness noise level variation with altitude in OASPL for several different true airspeeds. Depending on the true airspeed of the vehicle, thickness noise may either increase or decrease with increasing altitude. Higher true airspeeds correspond to higher advancing tip Mach numbers, where the thickness noise is more sensitive to further increases in advancing tip Mach number due to 


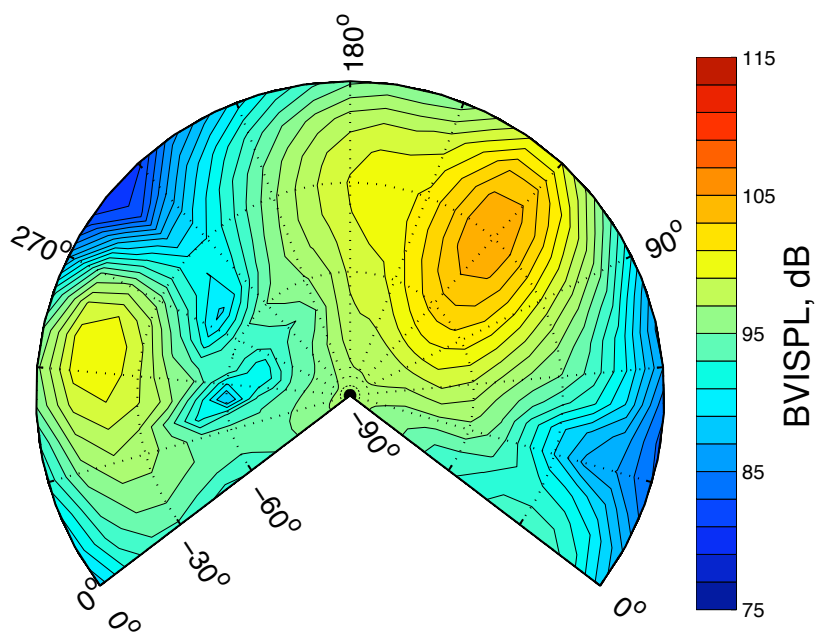

Fig. 34. 60kts TAS $-6^{\circ}$ descent BVISPL hemisphere at ISA 5,000 ft conditions.

$\left(\mu=0.14, \chi=0.048, C_{T}=0.0034, M_{H}=0.67\right)$

the decrease in ambient temperature. In practice, further increases in advancing tip Mach number at high flight speeds may yield even higher increases in noise than predicted as local transonic flow allows HSI noise to develop.

In practice, helicopters must be flown according to the indicated airspeed, because the aerodynamic forces and hence vehicle performance parameters are tied to the dynamic pressure, which varies with air density. However, so long as vehicle performance at altitude is taken into account when designing helicopter trajectories, helicopter noise modeling might be conducted more accurately on the basis of flight conditions defined by true airspeed than on the basis of indicated airspeed; in conjunction with the flight path angle, this is equivalent to specifying flight conditions on a non-dimensional basis of advance ratio and

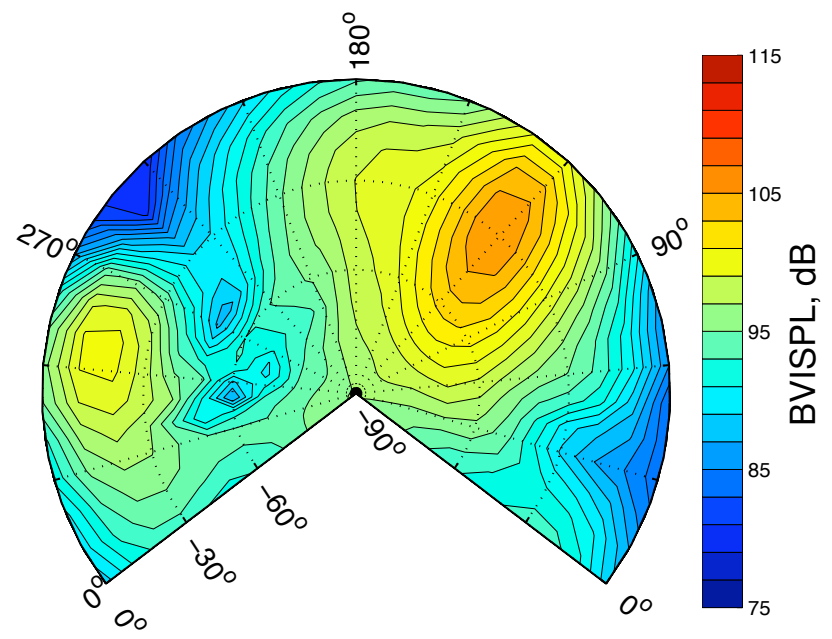

Fig. 35. 60kts TAS $-6^{\circ}$ descent BVISPL hemisphere at ISA $10,000 \mathrm{ft}$ conditions.

$\left(\mu=0.14, \chi=0.054, C_{T}=0.0039, M_{H}=0.68\right)$

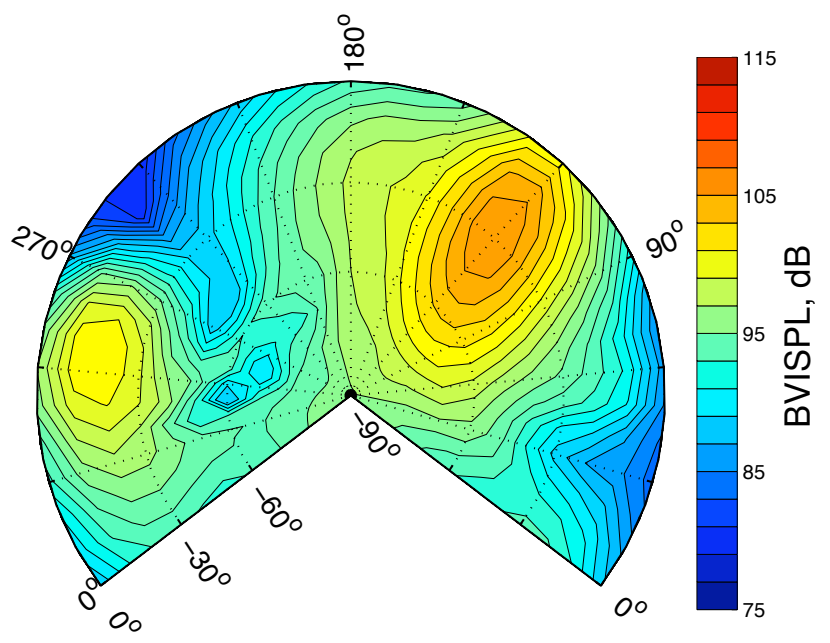

Fig. 36. 60kts TAS $-6^{\circ}$ descent BVISPL hemisphere at ISA 15,000 ft conditions.

$\left(\mu=0.14, \chi=0.064, C_{T}=0.0046, M_{H}=0.70\right)$

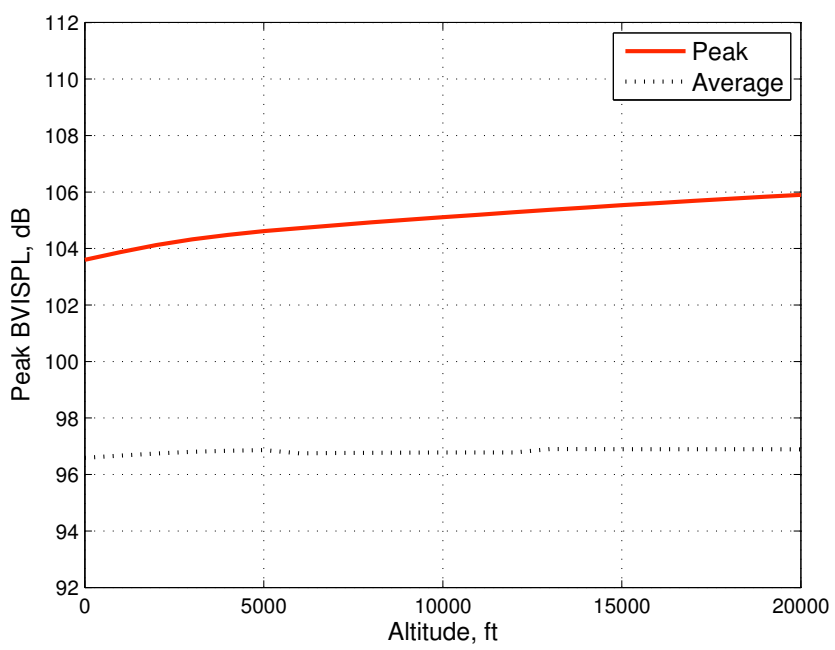

Fig. 37. Variation of BVISPL values for ISA altitude conditions at $60 \mathrm{kts}$ TAS, $-6^{\circ}$ descent flight.

wake skew ratio for steady flight conditions. Due to the strong effect of advance ratio on rotor harmonic noise, it is also cautioned that flight conditions should not be defined on the basis of ground speed in helicopter source noise models, since ground speed is not equivalent to true airspeed except in the absence of wind.

\section{Acknowledgements}

The authors would like to thank Dr. Ben Wel-C Sim and Michael E. Watts for helpful discussions during the preparation of this paper. In addition, the authors would like to thank Richard D. Sickenberger, David A. Conner, Charles D. Smith, Ernesto Moralez, and William A. Decker for their important roles in the 2006 flight test of the Bell 206B3 helicopter at Moffett Field, CA. 


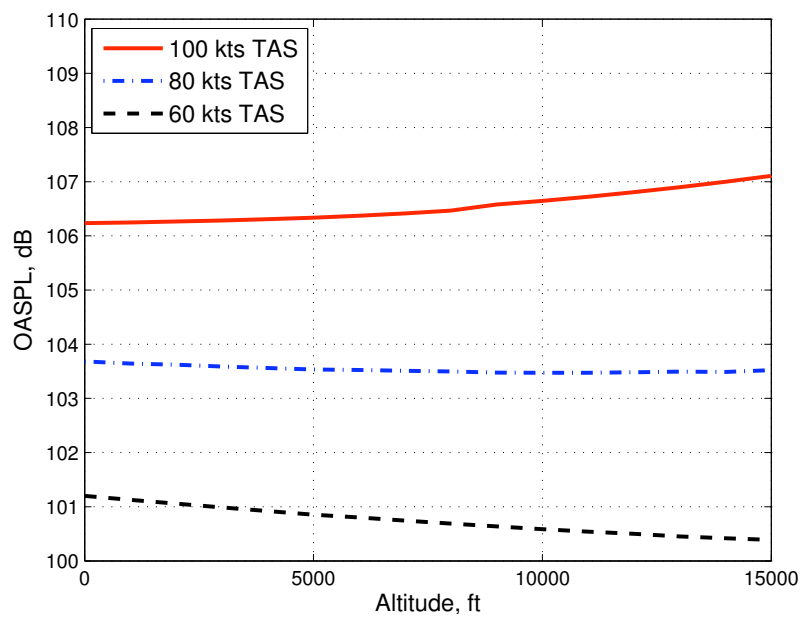

Fig. 38. Peak OASPL thickness noise level variation for ISA altitude conditions for constant TAS flight.

\section{References}

${ }^{1}$ Fleming, G. G. and Rickley, E. J., "Heliport Noise Model, HNM. Version 2.2 (User's Guide)," Technical report, Federal Aviation Administration, February 1994.

${ }^{2}$ Connor, T. L., "Integrated Noise Model-The Federal Aviation Administration's computer program for predicting noise exposure around an airport," Inter-noise 80: Noise control for the 80's, Jan 1980.

${ }^{3}$ Lucas, M. J. and Marcolini, M. A., "Rotorcraft Noise Model," AHS Technical Specialists' Meeting for Rotorcraft Acoustics and Aerodynamics, October 1997.

${ }^{4}$ Conner, D. A. and Page, J. A., "A Tool for Low Noise Procedures Design and Community Noise Impact Assessment: The Rotorcraft Noise Model (RNM)," Heli Japan, 2002.

${ }^{5}$ Browne, R. W., Munt, R. M., Simpson, C. R., and Williams, T., "Prediction of Helicopter Noise Contours for Land Use Planning," 10th AIAA/CEAS Aeroacoustics Conference, 2004.

${ }^{6}$ Gervais, M., Gareton, V., Dummel, A., and Heger, R., "Validation of EC130 and EC135 Environmental Impact Assessment using HELENA," American Helicopter Society 66th Annual Forum, May 2010.

${ }^{7}$ Guntzer, F., Spiegel, P., and Lummer, M., "Genetic Optimizations of EC-135 Noise Abatement Flight Procedures using and Aeroacoustic Database," 35th European Rotorcraft Forum, September 2009.

${ }^{8}$ Fleming, G. G., Plotkin, K. J., Roof, C. J., Ikelheimer, B. J., and Senzig, D. A., "Assessment of Tools for Modeling Aircraft Noise in the National Parks," Technical report, FICAN, March 2005.
${ }^{9}$ International Organization for Standardization, "Standard Atmosphere," Technical Report 2533:1975, ISO, 1975.

${ }^{10}$ Greenwood, E. and Schmitz, F. H., "A Parameter Identification Method for Helicopter Noise Source Identification and Physics-Based Semi-Empirical Modeling," American Helicopter Society 66th Annual Forum, May 2010.

${ }^{11}$ Greenwood, E. and Schmitz, F. H., "Separation of Main and Tail Rotor noise Ground-Based Acoustic Measurements using Time-Domain De-Dopplerization," 35th European Rotorcraft Forum, September 2009.

${ }^{12}$ Schmitz, F. H., Greenwood, E., Sickenberger, R. D., Gopalan, G., Sim, B. W.-C., Conner, D. A., Moralez, E., and Decker, W., "Measurement and Characterization of Helicopter Noise in Steady-State and Maneuvering Flight," American Helicopter Society 63rd Annual Forum, May 2007.

${ }^{13}$ Schmitz, F. H., Boxwell, D. A., Léwy, S., and Dahan, C., "Model- to Full-Scale Comparisons of Helicopter BladeVortex Interaction Noise," Journal of the American Helicopter Society, Vol. 29, (2), 1984, pp. 16-25.

doi: $10.4050 / J A H S .29 .16$

${ }^{14}$ Farassat, F., "Derivation of Formulations 1 and 1A of Farassat," Technical Report TM-2007-214853, NASA, 2007.

${ }^{15}$ Beddoes, T. S., "A wake model for high resolution airloads," International Conference on Rotorcraft Basic Research, February 1985.

${ }^{16}$ van der Wall, B. G., "The Effect of HHC on the Vortex Convection in the Wake of a Helicopter Rotor," Aerospace Science and Technology, Vol. 4, (5), 2000, pp. 321-336.

${ }^{17}$ Bhagwat, M. J. and Leishman, J. G., "Generalized Viscous Vortex Core Models for Application to Free-Vortex Wake and Aeroacoustic Calculations," 58th Annual Forum of the American Helicopter Society, June 2002.

${ }^{18}$ Landgrebe, A. J., "The wake geometry of a hovering helicopter rotor and its influence on rotor performance," Journal of the American Helicopter Society, Vol. 17, (4), 1972.

${ }^{19}$ Beddoes, T. S., "Practical Computation of Unsteady Lift," Vertica, Vol. 8, (1), 1984.

${ }^{20}$ Leishman, J. G., Principles of Helicopter Aerodynamics, Cambridge University Press, New York, second edition, 2006.

${ }^{21}$ Schmitz, F. H. and Sim, B. W.-C., "Radiation and Directionality Characteristics of Helicopter Blade-Vortex Interaction Noise," Journal of the American Helicopter Society, Vol. 48, (4), 2003, pp. 253-269. 
${ }^{22}$ Snyder, J. P., "Map Projections: A Working Manual," Technical Report PP1395, USGS, 1982.

${ }^{23}$ Ffowcs Williams, J. E. and Hawkings, D. L., "Sound generation by turbulence and surfaces in arbitrary motion," Transactions for the Royal Society of London, Vol. 264, May 1969, pp. 321-342.

${ }^{24}$ Schmitz, F. H. and Sim, B. W.-C., "Acoustic Phasing, Directionality and Amplification Effects of Helicopter BladeVortex Interactions," Journal of the American Helicopter Society, Vol. 46, (4), 2001, pp. 273-282.

doi: 10.4050/JAHS.46.273

${ }^{25}$ Schmitz, F. H., "Rotor Noise," Aeroacoustics of Flight Vehicles: Theory and Practice, edited by H. H. Hubbard, Vol. 1, Acoustical Society of America, first edition, 1995, pp. 65-145.

${ }^{26}$ Harris, F. D., "Rotary Wing Aerodynamics-Historical Perspective and Important Issues," AHS Technical Specialists' Meeting on Aerodynamics and Aeroacoustics, February 1987. 